\title{
JET SCHEMES AND INVARIANT THEORY
}

\author{
ANDREW R. LINSHAW, GERALD W. SCHWARZ, AND BAILIN SONG
}

\begin{abstract}
Let $G$ be a complex reductive group and $V$ a $G$-module. Then the $m$ th jet scheme $G_{m}$ acts on the $m$ th jet scheme $V_{m}$ for all $m \geq 0$. We are interested in the invariant ring $\mathcal{O}\left(V_{m}\right)^{G_{m}}$ and whether the map $p_{m}^{*}: \mathcal{O}\left((V / / G)_{m}\right) \rightarrow \mathcal{O}\left(V_{m}\right)^{G_{m}}$ induced by the categorical quotient map $p: V \rightarrow V / / G$ is an isomorphism, surjective, or neither. Using Luna's slice theorem, we give criteria for $p_{m}^{*}$ to be an isomorphism for all $m$, and we prove this when $G=\mathrm{SL}_{n}, \mathrm{GL}_{n}, \mathrm{SO}_{n}$, or $\mathrm{Sp}_{2 n}$ and $V$ is a sum of copies of the standard module and its dual, such that $V / / G$ is smooth or a complete intersection. We classify all representations of $\mathbb{C}^{*}$ for which $p_{\infty}^{*}$ is surjective or an isomorphism. Finally, we give examples where $p_{m}^{*}$ is surjective for $m=\infty$ but not for finite $m$, and where it is surjective but not injective.
\end{abstract}

\section{INTRODUCTION}

Given an irreducible scheme $X$ of finite type over an algebraically closed field $k$, the first jet scheme $X_{1}$ is just the total tangent space of $X$. For $m>1$, the $m^{\text {th }}$ jet scheme $X_{m}$ is a higher-order generalization that is determined by its functor of points. For every $k$-algebra $A$, we have a bijection

$$
\operatorname{Hom}\left(\operatorname{Spec}(A), X_{m}\right) \cong \operatorname{Hom}\left(\operatorname{Spec}\left(A[t] /\left\langle t^{m+1}\right\rangle\right), X\right) .
$$

When $X$ is nonsingular, $X_{m}$ is irreducible for all $m \geq 1$, and is an affine bundle over $X$ with fiber an affine space of dimension $m \operatorname{dim}(X)$. If $X$ is singular, the jet schemes are much more subtle and carry information about the singularities of $X$. The structural properties of $X_{m}$ are of interest, in particular the question of when $X_{m}$ is irreducible for all $m$. Mustata has shown that this holds when $X$ is locally a complete intersection with rational singularities, although these are not necessary conditions [Mu].

There are projections $X_{m+1} \rightarrow X_{m}$, and the arc space is defined to be

$$
X_{\infty}=\lim _{\leftarrow} X_{m} .
$$

Even though it is generally not of finite type, $X_{\infty}$ has some nicer properties than $X_{m}$; for example, it is always irreducible [Kol]. Arc spaces were originally studied by Nash in an influential paper [Na], in which he asked whether there is a bijection between the irreducible components of $X_{\infty}$ lying over the singular locus of $X$, and the essential divisors over $X$. This question is known as the Nash problem. It has been answered affirmatively for many classes of singular varieties, although counterexamples are known [IK]. Arc spaces are also important in Kontsevich's theory of motivic integration [Kon]. Given a complex algebraic variety $X$ and a resolution of singularities $Y \rightarrow X$ such that the discrepancy divisor $D$ has simple normal crossings, the motivic integral of $X$ is the integral

2010 Mathematics Subject Classification. 13A50,14L24,14L30.

Key words and phrases. jet schemes, classical invariant theory.

The authors thank Peter Littelmann for useful conversations. 
of a certain function $F_{D}$ defined on the arc space $Y_{\infty}$, with respect to a measure on $Y_{\infty}$. Unlike ordinary integration, this measure takes values not in $\mathbb{R}$, but in a certain completion of the Grothendieck ring of algebraic varieties. Motivic integration was originally used by Kontsevich to prove that birationally equivalent Calabi-Yau manifolds have the same Hodge numbers. This theory was subsequently developed by many authors including Batyrev, Denef, Loeser, Looijenga, Craw, and Veys [Bat][DLI] [Loo][Cr] [Ve]. A survey of these ideas and some of their applications can be found in [DLII].

Our goal in this paper is to establish some foundational results on the interaction between jet schemes, arc spaces, and classical invariant theory. If $G$ is a complex reductive group, $G_{m}$ is an algebraic group which is a unipotent extension of $G$. Let $Y$ be an affine $G$-variety and let $p: Y \rightarrow Y / / G=\operatorname{Spec}\left(\mathcal{O}(Y)^{G}\right)$ be the categorical quotient. Then $p$ induces a morphism $p_{m}: Y_{m} \rightarrow(Y / / G)_{m}$ and a homomorphism

$$
p_{m}^{*}: \mathcal{O}\left((Y / / G)_{m}\right) \rightarrow \mathcal{O}\left(Y_{m}\right)^{G_{m}}
$$

which was studied in some special cases by Eck in [E] and by Frenkel-Eisenbud in the appendix of [Mu]. We will find criteria for when this map is an isomorphism, surjective, or neither. First, using Luna's slice theorem, we show that all of these are local conditions (see Corollary 3.3). We are most interested in the case where $Y$ is a $G$-module $V$. Under mild hypotheses (see Corollary 3.20), we show that when $V / / G$ is smooth, $p_{m}^{*}$ is an isomorphism for all $m$. In Section 4 , we give a more refined criterion for $p_{m}^{*}$ to be an isomorphism for all $m$ (see Theorem 4.3) and we show that it holds when $G=\mathrm{SL}_{n}, \mathrm{GL}_{n}$, $\mathrm{SO}_{n}$, or $\mathrm{Sp}_{2 n}$ and $V$ is a sum of copies of the standard representation and its dual, such that $V / / G$ is a complete intersection. In Section 5, we consider representations of $\mathbb{C}^{*}$. Using techniques of standard monomial theory, we classify all cases where $p_{\infty}^{*}$ is surjective, and we show that $p_{\infty}^{*}$ is an isomorphism whenever it is surjective. In Section 6, we show that for $G=\mathrm{SL}_{n}$ and $V=\ell \mathbb{C}^{n}, p_{\infty}^{*}$ is surjective, even though $p_{m}^{*}$ generally fails to be surjective for finite values of $m$. For $n=2, p_{\infty}^{*}$ is injective, but it is not injective for $n \geq 3$. The question of whether $p_{\infty}^{*}$ is surjective for arbitrary representations $V=k \mathbb{C}^{n} \oplus \ell\left(\mathbb{C}^{n}\right)^{*}$ of $\mathrm{SL}_{n}$, and similar questions for the other classical groups, remain open.

Note that $\mathcal{O}\left(V_{\infty}\right)^{G_{\infty}}$ is finitely generated as a differential algebra whenever $p_{\infty}^{*}$ is surjective, since $\mathcal{O}\left((V / / G)_{\infty}\right)$ is generated by $\mathcal{O}(V / / G)$ as a differential algebra. An interesting problem is to find sufficient conditions for $\mathcal{O}\left(V_{\infty}\right)^{G_{\infty}}$ to be finitely generated as a differential algebra even if $p_{\infty}^{*}$ is not surjective. There are currently no examples where this is known to occur. Computer experiments suggest that this is the case for $G=\mathbb{C}^{*}$ and $V=\mathbb{C}^{2}$ with weights 2 and -3 (see Example 3.10).

Our results have a number of applications to the theory of vertex algebras that appear in separate papers. Vertex algebras are a class of nonassociative, noncommutative algebras that arose out of conformal field theory in the 1980s, and in the work of Borcherds [Bor] on the Moonshine conjecture. They were developed mathematically from several different points of view in the literature [BD][FBZ] [FLM $][\mathrm{Ka}]$. An abelian vertex algebra is just a commutative ring equipped with a derivation. For any variety $X$, the $\operatorname{ring} \mathcal{O}\left(X_{\infty}\right)$ has a derivation $D$ which makes it an abelian vertex algebra. On the other hand, many nonabelian vertex algebras $\mathcal{A}$ possess filtrations for which the associated graded algebra $\operatorname{gr}(\mathcal{A})$ is abelian and can be interpreted as $\mathcal{O}\left(X_{\infty}\right)$ for some $X$.

The first application of our results is to the commutant problem. Given a vertex algebra $\mathcal{V}$ and a subalgebra $\mathcal{A} \subset \mathcal{V}$, the $\operatorname{commutant} \operatorname{Com}(\mathcal{A}, \mathcal{V})$ is the subalgebra of $\mathcal{V}$ that commutes 
with $\mathcal{A}$. In [LSS], interesting examples of commutants were described using the fact that $\operatorname{gr}(\operatorname{Com}(\mathcal{A}, \mathcal{V}))$ is isomorphic to $\mathcal{O}\left((V / / G)_{\infty}\right)$ for a certain choice of $V$ and $G$. This leads to vertex algebra analogues of the classical Howe pairs of types $\mathrm{GL}_{n}-\mathrm{GL}_{m}, \mathrm{SO}_{n}-\mathfrak{s p}_{2 m}$, and $\mathrm{Sp}_{2 n}-\mathfrak{s o}_{2 m}$. The second application of our results is to the chiral de Rham complex [MSV]. This is a sheaf of vertex algebras on any nonsingular variety or complex manifold $X$ that contains the ordinary de Rham sheaf at weight zero, and captures stringy invariants of $X$ such as the elliptic genus. Using the fact that $p_{\infty}^{*}$ is an isomorphism for $G=\mathrm{SL}_{2}$ and $V=\ell \mathbb{C}^{2}$, Song gave a complete description of the global section algebra when $X$ is a Kummer surface; it is isomorphic to the $N=4$ superconformal algebra with $c=6$ [So]. Previously, the only nontrivial case where a description was known was $\mathbb{C P}^{n}[\mathrm{MS}]$.

\section{JET SCHEMES}

Throughout this paper our base field will be $\mathbb{C}$. We recall some basic facts about jet schemes, following the notation in [EM]. Let $X$ be an irreducible scheme of finite type. For each integer $m \geq 0$, the jet scheme $X_{m}$ is determined by its functor of points: for every $\mathbb{C}$-algebra $A$, we have a bijection

$$
\operatorname{Hom}\left(\operatorname{Spec}(A), X_{m}\right) \cong \operatorname{Hom}\left(\operatorname{Spec}\left(A[t] /\left\langle t^{m+1}\right\rangle\right), X\right) .
$$

Thus the $\mathbb{C}$-valued points of $X_{m}$ correspond to the $\mathbb{C}[t] /\left\langle t^{m+1}\right\rangle$-valued points of $X$. If $p>m$, we have projections $\pi_{p, m}: X_{p} \rightarrow X_{m}$ and $\pi_{p, m} \circ \pi_{q, p}=\pi_{q, m}$ when $q>p>m$. Clearly $X_{0}=X$ and $X_{1}$ is the total tangent space $\operatorname{Spec}\left(\operatorname{Sym}\left(\Omega_{X / \mathbb{C}}\right)\right)$. The assignment $X \mapsto X_{m}$ is functorial, and a morphism $f: X \rightarrow Y$ induces $f_{m}: X_{m} \rightarrow Y_{m}$ for all $m \geq 1$. If $X$ is nonsingular, $X_{m}$ is irreducible and nonsingular for all $\mathrm{m}$. Moreover, if $X, Y$ are nonsingular and $f: X \rightarrow Y$ is a smooth surjection, $f_{m}$ is surjective for all $m$.

If $X=\operatorname{Spec}(R)$ where $R=\mathbb{C}\left[y_{1}, \ldots, y_{r}\right] /\left\langle f_{1}, \ldots, f_{k}\right\rangle$, we can find explicit equations for $X_{m}$. Define new variables $y_{j}^{(i)}$ for $i=0, \ldots, m$, and define a derivation $D$ by $D\left(y_{j}^{(i)}\right)=$ $y_{j}^{(i+1)}$ for $i<m$, and $D\left(y_{j}^{(m)}\right)=0$, which specifies its action on all of $\mathbb{C}\left[y_{1}^{(0)}, \ldots, y_{r}^{(m)}\right]$. In particular, $f_{\ell}^{(i)}=D^{i}\left(f_{\ell}\right)$ is a well-defined polynomial in $\mathbb{C}\left[y_{1}^{(0)}, \ldots, y_{r}^{(m)}\right]$. Letting $R_{m}=$ $\mathbb{C}\left[y_{1}^{(0)}, \ldots, y_{r}^{(m)}\right] /\left\langle f_{1}^{(0)}, \ldots, f_{k}^{(m)}\right\rangle$, we have $X_{m} \cong \operatorname{Spec}\left(R_{m}\right)$. By identifying $y_{j}$ with $y_{j}^{(0)}$, we see that $R$ is naturally a subalgebra of $R_{m}$. There is a $\mathbb{Z}_{\geq 0}$-grading $R_{m}=\bigoplus_{n \geq 0} R_{m}[n]$ by weight, defined by wt $\left(y_{j}^{(i)}\right)=i$. For all $m, R_{m}[0]=R$ and $R_{m}[n]$ is an $R$-module.

Given a scheme $X$, define

$$
X_{\infty}=\lim _{\leftarrow} X_{m}
$$

which is known as the arc space of $X$. For a $\mathbb{C}$-algebra $A$, we have a bijection $\operatorname{Hom}\left(\operatorname{Spec}(A), X_{\infty}\right) \cong$ $\operatorname{Hom}(\operatorname{Spec} A[[t]], X)$. We denote by $\psi_{m}$ the natural map $X_{\infty} \rightarrow X_{m}$. If $X=\operatorname{Spec}(R)$ as above,

$$
X_{\infty} \cong \operatorname{Spec}\left(R_{\infty}\right), \text { where } R_{\infty}=\mathbb{C}\left[y_{1}^{(0)}, \ldots, y_{j}^{(i)}, \ldots\right] /\left\langle f_{1}^{(0)}, \ldots, f_{\ell}^{(i)}, \ldots\right\rangle .
$$

Here $i=0,1,2, \ldots$ and $D\left(y_{j}^{(i)}\right)=y_{j}^{(i+1)}$ for all $i$. By a theorem of Kolchin $[$ Kol $], X_{\infty}$ is irreducible whenever $X$ is irreducible.

\section{GROUP ACTIONS ON JET SCHEMES}

We establish some elementary properties of jet schemes and quotient mappings for reductive group actions. Mainly we see what one can say using Luna's slice theorem 
[Lun]. Let $G$ be a complex reductive algebraic group with Lie algebra $\mathfrak{g}$. For $m \geq 1$, $G_{m}$ is an algebraic group which is the semidirect product of $G$ with a unipotent group $U_{m}$. The Lie algebra of $G_{m}$ is $\mathfrak{g}[t] / t^{m+1}$. Given an affine $G$-variety $Y$, there is the quotient $Z:=Y / / G=\operatorname{Spec}\left(\mathcal{O}(Y)^{G}\right)$ and the canonical map $p: Y \rightarrow Z$ (sometimes denoted $p_{Y}$ ) which is dual to the inclusion $\mathcal{O}(Y)^{G} \subset \mathcal{O}(Y)$. We have a natural action of $G_{m}$ on $Y_{m}$, and we are interested in the invariant ring $\mathcal{O}\left(Y_{m}\right)^{G_{m}}$, the morphism $p_{m}: Y_{m} \rightarrow Z_{m}$ and whether $p_{m}^{*}: \mathcal{O}\left(Z_{m}\right) \rightarrow \mathcal{O}\left(Y_{m}\right)^{G_{m}}$ is an isomorphism, surjective, or neither. If $\varphi: X \rightarrow Y$ is a morphism of affine $G$-varieties, then $\varphi / / G$ will denote the induced mapping of $X / / G$ to $Y / / G$.

Recall that a morphism of varieties is étale if it is smooth with fibers of dimension zero. If $\varphi: X \rightarrow Y$ is a morphism where $X$ and $Y$ are smooth, then $\varphi$ is étale if and only if $d \varphi_{x}: T_{x} X \rightarrow T_{\varphi(x)} Y$ is an isomorphism for all $x \in X$.

Definition 3.1. Let $G$ be a reductive complex algebraic group and let $\varphi: X \rightarrow Y$ be an equivariant map of affine $G$-varieties. We say that $\varphi$ is excellent if the following hold.

(1) $\varphi$ is étale.

(2) $\varphi / / G: X / / G \rightarrow Y / / G$ is étale.

(3) The canonical map $\left(\varphi, p_{X}\right): X \rightarrow Y \times_{Y / / G} X / / G$ is an isomorphism.

Note that condition (1) is a consequence of conditions (2) and (3). Let us say that $X$ is $m$-very good if $p_{m}^{*}: \mathcal{O}\left((X / / G)_{m}\right) \rightarrow \mathcal{O}\left(X_{m}\right)^{G_{m}}$ is an isomorphism. We say that $X$ is $m$-good if $p_{m}^{*}$ is surjective, so that $\mathcal{O}\left(X_{m}\right)^{G_{m}}=p_{m}^{*} \mathcal{O}\left((X / / G)_{m}\right)$, and we say that $X$ is $m$-bad if $p_{m}^{*}$ is not surjective. Here $m$ is finite or $\infty$. Usually we drop the $m$. We say that $X$ is $D$-finite if $\mathcal{O}\left(X_{\infty}\right)^{G_{\infty}}$ is finitely generated as a differential algebra.

Lemma 3.2. Suppose that $\varphi: X \rightarrow Y$ is excellent. Then

(1) $X_{m} \simeq X / / G \times_{Y / / G} Y_{m}$.

(2) $(X / / G)_{m} \simeq X / / G \times_{Y / / G}(Y / / G)_{m}$.

If $Y$ is very good (resp. good or D-finite), then so is $X$, and conversely if $\varphi$ is surjective.

Proof. Since $\varphi$ is étale, $X_{m} \simeq X \times_{Y} Y_{m}$. Since $\varphi$ is excellent,

$$
X \times_{Y} Y_{m} \simeq X / / G \times_{Y / / G} Y \times_{Y} Y_{m} \simeq X / / G \times_{Y / / G} Y_{m}
$$

and since $\varphi / / G$ is étale, $(X / / G)_{m} \simeq X / / G \times_{Y / / G}(Y / / G)_{m}$. Thus we have (1) and (2).

If $Y$ is very good, then by (1) and (2)

$$
\mathcal{O}\left(X_{m}\right)^{G_{m}} \simeq \mathcal{O}(X / / G) \otimes_{\mathcal{O}(Y / / G)} \mathcal{O}\left((Y / / G)_{m}\right) \simeq \mathcal{O}\left((X / / G)_{m}\right),
$$

hence $X$ is very good. Similarly, $Y$ good implies that $X$ is good.

Conversely, if $\varphi$ is surjective and $\mathcal{O}\left(Y_{m}\right)^{G_{m}} \neq p_{Y, m}^{*} \mathcal{O}\left((Y / / G)_{m}\right)$, then, since $\varphi / / G$ is faithfully flat, we have that

$$
\mathcal{O}(X / / G) \otimes_{\mathcal{O}(Y / / G)} \mathcal{O}\left(Y_{m}\right)^{G_{m}} \neq \mathcal{O}(X / / G) \otimes_{\mathcal{O}(Y / / G)} p_{Y, m}^{*} \mathcal{O}\left((Y / / G)_{m}\right)
$$

and hence that $\mathcal{O}\left(X_{m}\right)^{G_{m}} \neq p_{X, m}^{*} \mathcal{O}\left((X / / G)_{m}\right)$. Hence $Y$ is good if $X$ is good. The proof that $Y$ is very good if $X$ is very good is similar.

Now

$$
\mathcal{O}\left(X_{\infty}\right)^{G_{\infty}} \cong \mathcal{O}\left(Y_{\infty}\right)^{G_{\infty}} \otimes_{\mathcal{O}(Y)^{G}} \mathcal{O}(X)^{G} .
$$


Thus if $Y$ is $D$-finite, then clearly so is $X$. Conversely, assume that $X$ is $D$-finite and that $\varphi$ is surjective. Set $A:=\mathcal{O}\left(X_{\infty}\right)^{G_{\infty}}$. Then we have the weight grading $A=\oplus_{n \in \mathbb{N}} A_{n}$ where $A_{0}=\mathcal{O}(X)^{G}$. Let $B$ denote $\mathcal{O}\left(Y_{\infty}\right)^{G_{\infty}}$. Then $B$ is graded and the isomorphism $A \cong B \otimes_{\mathcal{O}(Y)^{G}} \mathcal{O}(X)^{G}$ is an isomorphism of graded rings. Let $f_{i} \otimes h_{i}$ be generators of $A \simeq B \otimes_{\mathcal{O}(Y)^{G}} \mathcal{O}(X)^{G}$ as differential graded algebras. We may assume that each $f_{i}$ has weight $n_{i}$ for some $n_{i} \in \mathbb{N}$. Let $p_{1}, \ldots, p_{d}$ be generators of $\mathcal{O}(X)^{G}$. Then $D p_{j}=\sum f_{i j} \otimes h_{i j}$ where the $h_{i j}$ are elements of $\mathcal{O}(X)^{G}$ and the $f_{i j}$ are in $B_{1}$. Now take the collection of elements $f_{i}$ and $f_{i j}$ in $B$. An induction argument shows that $D$ applied repeatedly to the elements $f_{i} \otimes h_{i}$ ends up in the $\mathcal{O}(X)^{G}$-submodule of $A$ generated by $D$ applied to products of the elements $f_{i}$ and $f_{i j}$. Since $\varphi$ is faithfully flat, this shows that the $B_{0}$-submodule of $B_{n}$ generated by the elements $f_{i}$ and $f_{i j}$ is $B_{n}$ since this submodule tensored with $\mathcal{O}(X)^{G}$ is $A_{n}$. Hence the $f_{i}$ and $f_{i j}$ generate $B_{n}$ for all $n$ and $Y$ is $D$-finite.

A subset $S$ of $X$ is $G$-saturated if $S=p^{-1}(p(S))$; equivalently, $S$ is a union of fibers of $p$.

Corollary 3.3. $\quad$ (1) Suppose that $X=\cup X_{\alpha}$ where the $X_{\alpha}$ are Zariski open and $G$-saturated. Then $X$ is very good (resp. good or D-finite) if and only if each $X_{\alpha}$ is very good (resp. good or D-finite).

(2) Let $W$ be a $G$-module and $U=W_{f}$ where $f \in \mathcal{O}(W)^{G}$ and $f(0) \neq 0$. Then $W$ is very good (resp. good or D-finite) if and only $U$ is very good (resp. good or D-finite).

Proof. For (1) we may assume that we have a finite cover. Then the canonical map $\amalg X_{\alpha} \rightarrow$ $X$ is excellent and surjective and (1) follows from Lemma 3.2. For (2) we may assume that $U$ is very good (resp. good or $D$-finite). Now $W$ is the union of $U$ and finitely many translates $U_{\lambda}$ where $U_{\lambda}=\lambda \cdot U$ for $\lambda \in \mathbb{C}^{*}$. Clearly each $U_{\lambda}$ is very good (resp. good or $D$-finite) since $U$ is. Thus we can apply (1).

Let $H$ be a reductive subgroup of $G$ and $Y$ an affine $H$-variety. Then $G \times{ }^{H} Y$ denotes the quotient of $G \times Y$ by the $H$-action sending $(g, y)$ to $\left(g h^{-1}, h y\right)$ for $(g, y) \in G \times Y$ and $h \in H$. We denote the orbit of $(g, y)$ by $[g, y]$. We have an action of $G$ on the left on $G \times Y$ which commutes with the action of $H$ and induces a $G$-action on $G \times{ }^{H} Y$. Then $\left(G \times{ }^{H} Y\right) / / G \simeq Y / / H$. Note that $G \rightarrow G / H$ is a principal $H$-bundle, hence trivial over pullback via an étale surjective map to $G / H$.

Lemma 3.4. Let $H$ be a reductive subgroup of $G$ and $Y$ an affine $H$-variety. Then

$$
\mathcal{O}\left(\left(G \times{ }^{H} Y\right)_{m}\right)^{G_{m}} \simeq \mathcal{O}\left(Y_{m}\right)^{H_{m}} .
$$

Hence $G \times{ }^{H} Y$ is very good (resp. good or D-finite) if and only if $Y$ is very good (resp. good or $D$-finite).

Proof. For a trivial principal $H$ bundle $U \times H$, we have $(U \times H)_{m}=U_{m} \times H_{m}$ is a trivial $H_{m}$-bundle with quotient $U_{m}$. Thus $\left(G \times{ }^{H} Y\right)_{m}$ is the quotient of $G_{m} \times Y_{m}$ by the action of $H_{m}$ (it is a principal bundle).

Consider the action of $G_{m}$ on $G_{m} \times Y_{m}$. Then the quotient is clearly just projection to $Y_{m}$, so that $\mathcal{O}\left(G_{m} \times Y_{m}\right)^{G_{m}} \simeq \mathcal{O}\left(Y_{m}\right)$. Thus

$$
\mathcal{O}\left(G_{m} \times Y_{m}\right)^{G_{m} \times H_{m}} \simeq \mathcal{O}\left(Y_{m}\right)^{H_{m}}
$$

so that $\mathcal{O}\left(\left(G \times{ }^{H} Y\right)_{m}\right)^{G_{m}} \simeq \mathcal{O}\left(Y_{m}\right)^{H_{m}}$. 
Let $X$ be a smooth affine $G$-variety and suppose that $G x$ is a closed orbit. Then the isotropy group $H:=G_{x}$ is reductive and we have a splitting of $H$-modules $T_{x} X=$ $T_{x}(G x) \oplus N$. The representation $(N, H)$ is called the slice representation at $x$. Here is Luna's slice theorem [Lun] in our context.

Theorem 3.5. (1) There is a locally closed affine $H$-stable and $H$-saturated subvariety $S$ of $X$ containing $x$ such that $U:=G \cdot S$ is a $G$-saturated affine open subset of $X$. Moreover, the canonical G-morphism

$$
\varphi: G \times{ }^{H} S \rightarrow U, \quad[g, s] \mapsto g s
$$

is excellent.

(2) $S$ is smooth at $x$ and the $H$-modules $T_{x} S$ and $N$ are isomorphic. Possibly shrinking $S$ we can arrange that there is an excellent surjective $H$-morphism $\psi: S \rightarrow N_{f}$ which sends $x$ to 0 , inducing an excellent $G$-morphism

$$
\tau: G \times{ }^{H} S \rightarrow G \times{ }^{H} N_{f}
$$

where $f \in \mathcal{O}(N)^{H}$ and $f(0) \neq 0$.

Combining $3.2-3.5$ we obtain

Corollary 3.6. Suppose that $X$ is smooth. Let $(W, H)$ be a slice representation of $X$.

(1) If $X$ is very good (resp. good or D-finite) then so is $W$.

(2) If $W$ is very good (resp. good or D-finite) for each slice representation $(W, H)$ of $X$, then $X$ is very good (resp. good or D-finite).

From Lemma 3.2 we obtain

Corollary 3.7. Suppose that $X$ is smooth and that $f \in \mathcal{O}(X)^{G}$.

(1) If the slice representations of $X_{f}$ are very good, then

$$
\left(\mathcal{O}\left(X_{m}\right)^{G_{m}}\right)_{f} \simeq \mathcal{O}\left((X / / G)_{m}\right)_{f} .
$$

(2) If the slice representations of $X_{f}$ are good, then

$$
\left(\mathcal{O}\left(X_{m}\right)^{G_{m}}\right)_{f} \simeq\left(p_{m}^{*} \mathcal{O}\left((X / / G)_{m}\right)\right)_{f} .
$$

Our main focus in this paper will be on the case where $X$ is a finite-dimensional $G$ module $V$. Choose a basis $\left\{x_{1}, \ldots, x_{n}\right\}$ for $V^{*}$, so that

$$
\mathcal{O}(V) \cong \mathbb{C}\left[x_{1}, \ldots, x_{n}\right], \quad \mathcal{O}\left(V_{m}\right)=\mathbb{C}\left[x_{1}^{(i)}, \ldots, x_{n}^{(i)}\right], \quad 0 \leq i \leq m .
$$

The action of $G_{m}$ on $V_{m}$ induces the following action of its Lie algebra $\mathfrak{g}[t] / t^{m+1}$ on $\mathcal{O}\left(V_{m}\right)$. For $\xi \in \mathfrak{g}$,

$$
\xi t^{r}\left(x_{j}^{(i)}\right)=\lambda_{i}^{r}\left(\xi\left(x_{j}\right)\right)^{(i-r)}, \quad \lambda_{i}^{r}=\left\{\begin{array}{cc}
\frac{i !}{(i-r) !} & 0 \leq r \leq i \\
0 & r>i
\end{array} .\right.
$$

The invariant ring $\mathcal{O}\left(V_{m}\right)^{\mathrm{g}[t] / t^{m+1}}$ coincides with $\mathcal{O}\left(V_{m}\right)^{G_{m}}$ when $G$ is connected.

Lemma 3.8. Suppose that $V \oplus W$ is a representation of $G$. If $W$ is bad (resp. not $D$-finite) then so is $V \oplus W$. 
Proof. If $W$ is bad there is a $G_{m}$ - invariant polynomial on $W_{m}$ which does not come from $\mathcal{O}\left((W / / G)_{m}\right)$. Then clearly it cannot come from an element of $\mathcal{O}\left(((V \oplus W) / / G)_{m}\right)$. Now minimal generators of $\mathcal{O}\left(V_{\infty} \oplus W_{\infty}\right)^{G_{\infty}}$ can clearly be chosen to be bihomogeneous in the variables of $V_{\infty}$ and $W_{\infty}$. Thus if $V \oplus W$ is $D$-finite, then so is $W$.

The results above say that a representation is bad (resp. not $D$-finite) if a subrepresentation or slice representation is bad (resp. not $D$-finite). Now let us consider some examples.

Example 3.9. Let $(V, G)=(\mathbb{C}, \pm 1)$. Then $V$ is bad. Let $z$ be a coordinate function on $V$. Then $V_{1}$ has coordinates $z=z^{(0)}$ and $z^{(1)}$. The invariants of $G=G_{1}$ are generated by $z^{2}, z z^{(1)}$ and $\left(z^{(1)}\right)^{2}$. The invariants coming from the quotient are $z^{2}$ and $2 z z^{(1)}$. If one goes to degree 2 , then from $\mathbb{C}\left[z^{2}\right]$ we get $z^{2}, 2 z z^{(1)}$ and $2\left(z^{(1)}\right)^{2}+2 z z^{(2)}$. But among the $G_{2}$-invariants we have $z^{2}, z z^{(1)}, z z^{(2)},\left(z^{(1)}\right)^{2}, z^{(1)} z^{(2)}$ and $\left(z^{(2)}\right)^{2}$. Things are only getting worse. See Theorem 3.13 for the general case.

Example 3.10. Let $G=\mathbb{C}^{*}$ and let $V=\mathbb{C}^{2}$ with weights 2 and -3 . Then $\mathcal{O}(V)^{G}$ is generated by $z=x^{3} y^{2}$, so that $V / / G \cong \mathbb{C}$. For $m=1, w:=(D(z))^{2} / z=x\left(3 y x^{(1)}+2 x y^{(1)}\right)^{2}$ is not a function on $(V / / G)_{1}$, but it is a $G_{1}$-invariant function on $V_{1}$. Hence $V$ is 1-bad. In fact, it is $m$-bad for any $m \geq 1$. See Theorem 3.15 for a generalization. Computer calculations suggest that $V$ is $D$-finite with generators $z$ and $w$. Thus this is likely an example where $V$ is bad yet $D$-finite.

Example 3.11. Let $G=\mathrm{SL}_{3}$ and let $V$ be the direct sum of 6 copies of the standard representation $\mathbb{C}^{3}$, with basis $\left\{x^{(a, 0)}, y^{(a, 0)}, z^{(a, 0)} \mid a=1, \ldots, 6\right\}$. The generators of $\mathcal{O}(V)^{G}$ are $3 \times 3$ determinants $[a b c]$ corresponding to a choice of three distinct indices $a, b, c \in\{1, \ldots, 6\}$. Let $x^{(a, 1)}=D x^{(a, 0)}$ and similarly for $y$ and $z$, and define

$$
f=\sum_{\sigma \in \mathfrak{S}_{6}} \operatorname{sgn}(\sigma) x^{(\sigma(1), 0)} y^{(\sigma(2), 0)} z^{(\sigma(3), 0)} x^{(\sigma(4), 1)} y^{(\sigma(5), 1)} z^{(\sigma(6), 1)},
$$

where $\sigma$ runs over the group $\mathfrak{S}_{6}$ of permutations of $\{1, \ldots, 6\}$. Note that $f$ has degree 6 and weight 3 and lies in $\mathcal{O}\left(V_{1}\right)^{G_{1}}$, but $f \notin p_{1}^{*} \mathcal{O}\left((V / / G)_{1}\right)$, since elements of $p_{1}^{*} \mathcal{O}\left((V / / G)_{1}\right)$ of degree 6 can have weight at most 2 . Hence $V$ is 1 -bad, and in fact it is $m$-bad for all finite $m \geq 1$. However, $f$ can be expressed (up to a constant multiple) in the form

$$
\sum_{\sigma \in \mathfrak{S}_{6}}[\sigma(1) \sigma(2) \sigma(3)]([\sigma(4) \sigma(5) \sigma(6)])^{(3)},
$$

so $f \in p_{3}^{*} \mathcal{O}\left((V / / G)_{3}\right)$. In Theorem 6.5 and Example6.6 below we show that $p_{\infty}^{*}$ is surjective but not injective.

Remark 3.12. The surjectivity of $p_{\infty}^{*}$ is equivalent to the condition that every element of $\mathcal{O}\left(V_{\infty}\right)^{G_{\infty}}$ of weight $m$ lies in $p_{m}^{*} \mathcal{O}\left((V / / G)_{m}\right)$.

Theorem 3.13. Suppose that $G \subset \mathrm{GL}(V)$ is finite and nontrivial. Then $V$ is $m$-bad for any $m \geq 1$ and $V$ is not D-finite.

Proof. Note that $G_{m}=G$ for all $m$. Let $k>0$ be minimal such that there is a homogeneous generator of $\mathcal{O}(V)^{G}$ of degree $k$. Let $f_{1}, \ldots, f_{\ell}$ be a basis of the generators of degree $k$. We have $V_{1} \simeq T V=V \oplus V^{\prime}$ where $V^{\prime}$ is a $G$-module isomorphic to $V$. Using the isomorphism we obtain minimal generators $f_{1}^{\prime}, \ldots, f_{\ell}^{\prime}$ of $\mathcal{O}\left(V^{\prime}\right)^{G}$ which are linearly independent. The $f_{i}^{\prime}$ exist in every $\mathcal{O}\left(V_{m}\right)^{G}$ for $m \geq 1$. They are not in $p_{m}^{*} \mathcal{O}\left((V / G)_{m}\right)$ because the only 
possibility is that $f_{i}^{\prime}=D^{k} f_{i}$ for all $i$ where the latter have terms involving the variables of $V_{k}$ not in $V_{1}$. Thus $V$ is $m$-bad for all $m \geq 1$.

Let $f$ be a homogeneous element of $\mathcal{O}\left(V_{\infty}\right)^{G}$ of minimal positive degree, say $m$. Let $1 \leq i_{1}<\cdots<i_{m} \leq s$. Then there is a polarization $f_{i_{1}, \ldots, i_{m}}$ which is multilinear and invariant on the copies of $V$ in $V_{s}$ corresponding to the indices $i_{1}, \ldots, i_{m}$. Now consider $i_{j}=r m+j$ for $r \geq 1$. If $f:=f_{i_{1}, \ldots, i_{m}}$ is in the differential subalgebra of $\mathcal{O}\left(V_{\infty}\right)^{G}$ generated by $\mathcal{O}\left(V_{r m}\right)^{G}$, then we must have that $f$ is a sum of $D$ to some powers applied to invariants of degree $m$ lying in $\mathcal{O}\left(V_{r m}\right)^{G}$. But it is easy to see that such a sum can never give $f$.

Corollary 3.14. If $X$ is a smooth affine $G$-variety and $(W, H)$ is a slice representation of $X$ with $H$ finite and acting nontrivially on $W$, then $X$ is bad and not $D$-finite.

We need some more background on the action of $G$, see [Lun]. The points of $V / / G$ are in one to one correspondence with the closed orbits $G v, v \in V$. Let $H:=G_{v}$ be the isotropy group (which is reductive) and let $(W, H)$ be the slice representation. The fiber $p^{-1}(p(v))$ is isomorphic to $G \times{ }^{H} \mathcal{N}(W)$ where $\mathcal{N}(W):=p_{W}^{-1}\left(p_{W}(0)\right)$ is the null cone of $W$. For the rest of this section, we set $Z:=V / / G$. Let $(H)$ denote the conjugacy class of $H$ in $G$ and let $Z_{(H)}$ denote the closed orbits $G v^{\prime}$ such that $G_{v^{\prime}} \in(H)$. Then there are finitely many strata $Z_{(H)}$ each of which is smooth and irreducible. For reductive subgroups $H_{1}$ and $H_{2}$ of $G$, write $\left(H_{1}\right) \leq\left(H_{2}\right)$ if $H_{1}$ is $G$-conjugate to a subgroup of $H_{2}$. Then among the isotropy classes of closed orbits, there is a unique minimum $(H)$ with respect to $\leq$, called the principal isotropy class. We also call $H$ a principal isotropy group and corresponding closed orbits are called principal orbits. Then $Z_{(H)}$ is the unique open stratum in $Z$ and we also denote it by $Z_{\mathrm{pr}}$. Let $G v$ be a principal orbit with $G_{v}=H$. Then the fiber of $p$ through $v$ is of the form $G \times{ }^{H} W$ where $W$ is the nontrivial part of the slice representation of $H$ at $v$ and $\mathcal{O}(W)^{H}=\mathbb{C}$. We say that a $G$-module is stable if the general $G$-orbit is closed. Then the slice representations of the principal isotropy groups are trivial and $V_{\mathrm{pr}}:=p^{-1}\left(Z_{\mathrm{pr}}\right)$ is open and consists of principal orbits.

Let $S$ be an irreducible hypersurface in $Z$. Then the ideal of $S$ is generated by an invariant $f$. Write $f=f_{1}^{a_{1}} \ldots f_{n}^{a_{n}}$ where the $f_{i}$ are irreducible polynomials in $\mathcal{O}(V)$. We say that the irreducible component $\left\{f_{i}=0\right\}$ of $p^{-1}(S)$ is schematically reduced if $a_{i}=1$. Equivalently, the differential $d f$ does not vanish at some point of $\left\{f_{i}=0\right\}$. We say that $p^{-1}(S)$ is schematically reduced if all of its irreducible components are. Equivalently, $f$ generates the ideal of $p^{-1}(S)$ in $\mathcal{O}(V)$. The codimension one strata of $V$ are the inverse images in $V$ of the codimension one strata of $Z$.

Theorem 3.15. Let $V$ be a $G$-module such that $\operatorname{dim} Z=1$. Then $Z \simeq \mathbb{C}$.

(1) If $\mathcal{O}\left(V_{m}\right)^{G_{m}}=p_{m}^{*} \mathcal{O}\left(\mathbb{C}_{m}\right)$ for some $m \geq 1$, then an irreducible component of $\mathcal{N}(V)$ is schematically reduced.

(2) If an irreducible component of $\mathcal{N}(V)$ is schematically reduced, then $\mathcal{O}\left(V_{m}\right)^{G_{m}}=p_{m}^{*} \mathcal{O}\left(\mathbb{C}_{m}\right)$ for all $m \geq 1$.

Proof. Since $Z$ is normal of dimension one, we have $Z \simeq \mathbb{C}$ and $\mathcal{O}(V)^{G}$ is generated by a homogeneous invariant $p$. Write $p=p_{1}^{a_{1}} \ldots p_{n}^{a_{n}}$ where the $p_{i}$ are irreducible polynomials in $\mathcal{O}(V)$. Suppose that no irreducible component of $\mathcal{N}(V)$ is schematically reduced. Then $a_{i} \geq 2$ for all $i$ and $(D p)^{2}$ is divisible by $p$, yet $(D p)^{2} / p$ is not the pullback of an element of $\mathcal{O}\left(\mathbb{C}_{m}\right)$ and we have (1). 
Now suppose that an irreducible component of $\mathcal{N}(V)$ is schematically reduced and that $V$ is stable. Let $V^{\prime}=\{v \in V \mid d p(v) \neq 0\}$. Then $V^{\prime}$ is $G$-stable, open and dense in $V$. Since $d p$ does not vanish somewhere on $\mathcal{N}(V), p$ is a smooth mapping of $V^{\prime}$ onto $\mathbb{C}$. Hence $p_{m}: V_{m}^{\prime} \rightarrow \mathbb{C}_{m}$ is smooth and surjective. The principal fibers of $p: V \rightarrow \mathbb{C}$ are homogeneous spaces $G / H$ where $H$ is reductive. Since $G \rightarrow G / H$ is a principal $H$-bundle, $G_{m} \rightarrow(G / H)_{m}$ is a principal $H_{m}$-bundle and $(G / H)_{m} \simeq G_{m} / H_{m}$. It follows that the fibers of $p_{m}$ in $\left(V_{\mathrm{pr}}\right)_{m}$ are homogeneous spaces $G_{m} / H_{m}$. Hence any $h \in \mathcal{O}\left(V_{m}\right)^{G_{m}}$ is the pullback of a rational function $\tilde{h}$ on $\mathbb{C}_{m}$. If $\tilde{h}$ has poles, then so does $p_{m}^{*} \tilde{h}=h$. Hence $\tilde{h}$ is in $\mathcal{O}\left(\mathbb{C}_{m}\right)$ and we have proved (2) in case $V$ is stable.

Now suppose that $V$ is not stable. Then the principal fibers are $G \times{ }^{H} W$ where $\mathcal{O}(W)^{H}=$ $\mathbb{C}$. Since $\mathcal{O}(W)^{H}=\mathbb{C}$, by Hilbert-Mumford there is a 1-parameter subgroup $\lambda: \mathbb{C}^{*} \rightarrow H$ such that $H W_{\lambda}=W$ where $W_{\lambda}$ is the sum of the strictly positive weight spaces of $\lambda$. There is a dense open subset $W_{\lambda}^{\prime}$ of $W_{\lambda}$ such that $H \times W_{\lambda}^{\prime} \rightarrow W^{\prime} \subset W$ is surjective and smooth where $W^{\prime}$ is open in $W$. Then $H_{m} \times W_{\lambda, m}^{\prime} \rightarrow W_{m}^{\prime}$ is surjective and smooth where $\lambda$ has only positive weights on $W_{\lambda, m}^{\prime}:=\left(W_{\lambda}^{\prime}\right)_{m}$. Hence the $H_{m}$-invariants of $W_{m}$ are just the constants. It follows that the $G_{m}$-invariants on $G_{m} \times{ }^{H_{m}} W_{m}$ are constants and the proof above goes through.

Remark 3.16. Whenever $Z_{m}$ is irreducible and reduced, $p_{m}^{*}$ is injective since $p_{m}$ is dominant. Hence good and very good are equivalent in this case.

Corollary 3.17 (Eck). Let $V$ be a stable $G$-module with $\operatorname{dim} Z=1$. Assume that the generating invariant $p$ is irreducible. Then $V$ is very good.

We say that the $G$-module $V$ is coregular if $Z$ is smooth. Equivalently, $\mathcal{O}(V)^{G}$ is a polynomial ring [Kr, II.4.3 Lemma 1]. In this case, good and and very good are equivalent by Remark 3.16,

Corollary 3.18. Let $V$ be coregular. Then $V$ is very good if and only if each codimension one stratum of $V$ has a schematically reduced irreducible component.

Proof. If a codimension one stratum has no schematically reduced irreducible component, then the corresponding slice representation is of the form $(W+\theta, H)$ where $\theta$ is a trivial representation, $W^{H}=0, \operatorname{dim} W / / H=1$ and $\mathcal{N}(W)$ has no schematically reduced irreducible component. Then Corollary 3.6 and Theorem 3.15 show that $V$ is bad.

Now assume that each codimension one stratum has a schematically reduced irreducible component. Let $V^{\prime}$ be the set of points of $V$ where $d p$ has maximal rank and let $Z^{\prime} \subset Z$ be the image. Then the complement of $Z^{\prime}$ has codimension at least 2 in $Z \simeq \mathbb{C}^{k}$. As in the case $k=1$, any $G_{m}$ - invariant polynomial on $V_{m}$ is the pullback of a rational function on $\left(\mathbb{C}^{k}\right)_{m}$ which has no poles on $Z_{m}^{\prime}$. But the complement of $Z_{m}^{\prime}$ in $\left(\mathbb{C}^{k}\right)_{m}$ has codimension at least 2 . Hence our $G_{m}$-invariant polynomial is the pullback of a polynomial on $\left(\mathbb{C}^{k}\right)_{m}$.

Now let $G$ be a connected complex reductive group and let $V$ be a $G$-module. We impose a mild technical condition which is automatic if $G$ is semisimple; we assume that $\mathcal{O}(V)$ contains no nontrivial one-dimensional invariant subspaces. Equivalently, we assume that every semi-invariant of $G$ is invariant.

Lemma 3.19. Assume that $G$ is connected and that every semi-invariant of $\mathcal{O}(V)$ is invariant. 
(1) A function $f \in \mathcal{O}(V)^{G}$ is irreducible in $\mathcal{O}(V)$ if and only if it is irreducible in $\mathcal{O}(V)^{G}$. In particular, $\mathcal{O}(V)^{G}$ is a UFD.

(2) The codimension one strata of $V$ are irreducible and schematically reduced.

(3) Let $S \subset V / / G$ have codimension at least 2. Let $f_{1}$ and $f_{2}$ be relatively prime elements of $\mathcal{O}(V)^{G}$ which vanish on $S$. Then $f_{1}$ and $f_{2}$ are relatively prime elements of $\mathcal{O}(V)$, hence $p^{-1}(S)$ has codimension at least 2 in $V$.

Proof. We only need to prove (1) since this implies both (2) and (3). Let $f \in \mathcal{O}(V)^{G}$ and let $f=p_{1} \cdots p_{k}$ be its prime factorization in $\mathcal{O}(V)$. Since every semi-invariant of $G$ is invariant, each $g \in G$ must permute the factors of $f$, so $f$ determines a homomorphism from $G$ to the permutation group on $k$ letters. But $G$ is connected and this map is continuous, so it must be trivial. Therefore each $p_{i} \in \mathcal{O}(V)^{G}$.

Corollary 3.20. Suppose that $V$ is coregular, $G$ is connected and every semi-invariant of $\mathcal{O}(V)$ is invariant. Then $V$ is very good.

As above, let $V^{\prime}$ be the set of points in $V$ where $d p$ has maximal rank, and let $Z^{\prime} \subset Z$ be the image of $V^{\prime}$.

Lemma 3.21. Assume that $G$ is connected and that every semi-invariant of $\mathcal{O}(V)$ is invariant. Let $S$ be a codimension one stratum of $Z$.

(1) The rank of $d p$ is $\operatorname{dim} Z$ on an open dense subset of $p^{-1}(S)$.

(2) $V \backslash V^{\prime}$ has codimension at least 2 in $V$.

Proof. Let $F=p^{-1}(p(v))$ where $G v$ is closed and $p(v)$ lies in $S$. Since $\mathcal{O}(V)^{G}$ is a UFD, the closure of $S$ is defined by an irreducible invariant $f$. Hence $p^{-1}(\bar{S})$ is irreducible and $d f \neq 0$ on an open dense subset of $p^{-1}(S)$. Now $F$ is isomorphic to $G \times{ }^{H} \mathcal{N}(W)$ where $W$ is the slice representation of $H=G_{v}$. This fiber is the same everywhere over $S$ and $p^{-1}(S)$ is a fiber bundle over $S$ with fiber $F$. Thus $f^{-1}(0)$ is schematically reduced if and only if $F$ is schematically reduced, i.e., the $G$-invariant polynomials vanishing at $p(v)$ generate the ideal of $F$ in $\mathcal{O}(V)$. Thus at a smooth point of $F$ the rank of $d p$ must be maximal. It follows that $d p$ has maximal rank on an open dense subset of $p^{-1}(S)$ and we have (1). By Lemma 3.19(3), if $T$ is a stratum of $Z$ where $\operatorname{codim}_{Z} T \geq 2$, then $\operatorname{codim}_{V} p^{-1}(T) \geq 2$. Hence (2) follows from (1).

Corollary 3.22. Let $(U, K)$ be a slice representation of $V$ and let $S=(U / / K)_{(H)}$ be a codimension one stratum where $H \subset K$. Then $p_{U}^{-1}(\bar{S})$ is schematically reduced.

Proof. Over a point of $S$, the schematic fiber of $p_{U}$ is $K \times{ }^{H} \mathcal{N}(W)$ where $W$ is the slice representation of $H$. The schematic fiber of $p_{V}$ is $G \times{ }^{H} \mathcal{N}(W)$ over points of $S^{\prime}:=(V / / G)_{(H)}$. Thus the ranks of $d p_{U}$ and $d p_{V}$ are the same on the inverse images of $S$ and $S^{\prime}$, respectively, and it follows that the hypersurface $p_{U}^{-1}(\bar{S})$ is schematically reduced.

Proposition 3.23. Let $H$ be reductive and $W$ an $H$-module such that the codimension one strata are schematically reduced. Set $Y:=W / / H$. Suppose that $W^{\prime} \cap \mathcal{N}(W) \neq \emptyset$. Then $W$ is coregular and very good, and $p_{W}\left(W^{\prime}\right)=Y$.

Proof. Since $d p_{W}$ has maximal rank at a point of $\mathcal{N}(W)$, the image point $0 \in Y$ is smooth. But $Y$ has a cone structure (induced by the scalar action of $\mathbb{C}^{*}$ on $W$ ). It follows that $Y$ is smooth, i.e., $W$ is a coregular representation of $H$. By Corollary 3.18 we have that 
$\mathcal{O}\left(W_{m}\right)^{H_{m}}=p_{W, m}^{*} \mathcal{O}\left(Y_{m}\right)$. Since $W^{\prime} \cap \mathcal{N}(W) \neq \emptyset, p_{W}\left(W^{\prime}\right)$ contains a neighborhood of $0 \in Y$. Since $W^{\prime}$ and $Y$ are cones, $p_{W}\left(W^{\prime}\right)=Y$.

Theorem 3.24. Assume that $G$ is connected and that every semi-invariant of $\mathcal{O}(V)$ is invariant. Then for all $m \geq 1$, we have $\mathcal{O}\left(V_{m}\right)^{G_{m}}=p_{m}^{*} \mathcal{O}\left(Z_{m}^{\prime}\right)$.

Proof. Let $G v$ be a closed orbit such that $p^{-1}(p(v))$ intersects $V^{\prime}$. Let $(W, H)$ be the slice representation at $v$. Then Proposition 3.23 and Corollary 3.22 show that $\mathcal{O}\left(W_{m}\right)^{H_{m}}=$ $p_{W, m}^{*} \mathcal{O}\left((W / / H)_{m}\right)$. Using the slice theorem we see that this implies that $\mathcal{O}\left(U_{m}\right)^{G_{m}}=$ $p_{m}^{*} \mathcal{O}\left(\tilde{Z}_{m}\right)$ where $U$ is a $G$-saturated neighborhood of $G v$ and $\tilde{Z}:=p(U)$ is a neighborhood of $p(v)$. Thus given $f \in \mathcal{O}\left(V_{m}^{\prime}\right)^{G_{m}}$ there is a unique $h \in \mathcal{O}\left(Z_{m}^{\prime}\right)$ such that $p_{m}^{*} h=f$ on $V_{m}^{\prime}$. Since $\operatorname{codim}_{V} V \backslash V^{\prime} \geq 2, f$ extends to an element of $\mathcal{O}\left(V_{m}\right)^{G_{m}}$.

\section{Classical RePresentations of Classical groups}

Let $G=\mathrm{SL}_{n}$ and $V=k \mathbb{C}^{n}+\ell\left(\mathbb{C}^{n}\right)^{*}$. For applications to vertex algebras it would be nice to show that $p_{\infty}^{*} \mathcal{O}\left(Z_{\infty}\right)=\mathcal{O}\left(V_{\infty}\right)^{G_{\infty}}$. But we don't know if this is true for general $k$ and $\ell$. On the positive side we are able to show that $p_{\infty}^{*}$ is surjective when $k$ or $\ell$ is zero or when $Z$ is a complete intersection. In this section we concentrate on the complete intersection case. We also handle the complete intersection classical representations of the other classical groups (with $\mathrm{O}_{n}$ excluded, since it is not connected).

Let $G$ be reductive and let $V=V_{1} \oplus V_{2}$ be a sum of $G$-modules. If $Z_{i}:=V_{i} / / G$ is not a complete intersection for some $i$, then neither is $Z=V / / G$. This is clear because the generators and relations of $\mathcal{O}(V)^{G}$ can be chosen to be bihomogeneous in the generators of $\mathcal{O}\left(V_{i}\right)$, so a minimal set of generators and relations for $\mathcal{O}(V)^{G}$ will contain minimal sets of generators and relations for each $\mathcal{O}\left(V_{i}\right)^{G}$.

Lemma 4.1. Suppose that $Z$ is a complete intersection. Then $W / / H$ is a complete intersection for every slice representation $(W, H)$ of $V$.

Proof. Let $y$ denote the image of $0 \in W$ in $W / / H$. By Luna's slice theorem, up to étale mappings (which preserve the property of being a complete intersection), we have an isomorphism of a neighborhood of $y$ with a neighborhood of some $z \in Z$. It follows that $W / / H$ is a complete intersection in a neighborhood of $y$. Let $p_{1}, \ldots, p_{d}$ be minimal homogeneous generators of $\mathcal{O}(W)^{H}$. Then their relations are minimally generated by polynomials $h_{j}\left(y_{1}, \ldots, y_{d}\right)$ which are homogeneous when we give $y_{i}$ the degree of $p_{i}$. Since $W / / H$ is a complete intersection near $y$, the number of $h_{j}$ is $d-\operatorname{dim} W / / H$. Hence $W / / H$ is a complete intersection.

Now consider $(V, G)=\left(k \mathbb{C}^{n}, \mathrm{SL}_{n}\right), n \geq 3$. If $k=n+2$, then $\mathcal{O}(V)^{G}$ has $\left(\begin{array}{c}n+2 \\ 2\end{array}\right)$ minimal generators and $\left(\begin{array}{c}n+2 \\ 4\end{array}\right)$ minimal relations. Its dimension is $2 n+1>\left(\begin{array}{c}n+2 \\ 2\end{array}\right)-\left(\begin{array}{c}n+2 \\ 4\end{array}\right)$, which shows that we don't have a complete intersection for $k \geq n+2$. We say that there are too many relations. For the case $n=2$ we don't have a complete intersection for $k \geq 5$ but we do for $k=4$. Note that here the representations of $\mathrm{SL}_{2}$ on $\mathbb{C}^{2}$ and $\left(\mathbb{C}^{2}\right)^{*}$ are isomorphic.

Let $H_{1}, \ldots, H_{r}$ be representatives for the conjugacy classes of isotropy groups of closed orbits in $V$. We say that $H_{i}$ is maximal proper if $H_{i} \neq G$ and every other $H_{j}$ besides $G$ is conjugate to an isotropy group of the slice representation of $H_{i}$. Of course, in general, such an isotropy group does not exist. 
Lemma 4.2. Let $(V, G)=\left(k \mathbb{C}^{n}+\ell\left(\mathbb{C}^{n}\right)^{*}, \mathrm{SL}_{n}\right), n \geq 3$ where $k \ell \neq 0$. Then the maximal proper isotropy group is $\mathrm{SL}_{n-1}$ with slice representation $(k-1) \mathbb{C}^{n-1}+(\ell-1)\left(\mathbb{C}^{n-1}\right)^{*}$ (up to trivial factors).

Proof. We may assume that $k \geq \ell$. Write $V=V_{1} \oplus \cdots \oplus V_{\ell} \oplus(k-\ell) \mathbb{C}^{n}$ where each $V_{i}$ is a copy of $\mathbb{C}^{n} \oplus\left(\mathbb{C}^{n}\right)^{*}$. Then [Sch, 3.8] implies that any proper isotropy class $(H)$ of $V$ is contained in a proper isotropy class $(L)$ of one of the $V_{i}$ or $(k-\ell) \mathbb{C}^{n}$. By [We, Theorem 2.6.A], the invariants of any $V_{i}$ are generated by the contraction of $\mathbb{C}^{n}$ with $\left(\mathbb{C}^{n}\right)^{*}$. The nonzero orbits where the invariant does not vanish are closed and have isotropy class $\left(\mathrm{SL}_{n-1}\right)$. Again by [We, Theorem 2.6.A] the nonconstant invariants of $(k-\ell) \mathbb{C}^{n}$ are generated by determinants (this only happens for $k-\ell \geq n$ ) in which case the orbits where a determinant does not vanish are closed and have trivial isotropy groups. Hence $\mathrm{SL}_{n-1}$ is maximal proper. Let $W$ be the slice representation of $\mathrm{SL}_{n-1}$. Then we have that

$$
W \oplus \mathfrak{s} l_{n} / \mathfrak{s} l_{n-1}=V \text { as } \mathrm{SL}_{n-1} \text {-modules. }
$$

This gives that $W=(k-1) \mathbb{C}^{n-1}+(\ell-1)\left(\mathbb{C}^{n-1}\right)^{*}$ (up to trivial factors).

Now suppose that $(V, G)$ is as in the lemma above and that $k=n+1, n \geq 3$, and that $Z$ is a complete intersection. If $\ell=n$, then there are again too many relations. Thus we must have $\ell<n$. In fact we can have $\ell=(n-1)$. Consider the action of $\mathrm{SL}_{n+1}$ on $\operatorname{Hom}\left(\mathbb{C}^{n+1}, \mathbb{C}^{n}\right) \simeq(n+1) \mathbb{C}^{n}$ and $\mathrm{SL}_{n-1}$ on $\operatorname{Hom}\left(\mathbb{C}^{n-1},\left(\mathbb{C}^{n}\right)^{*}\right) \simeq(n-1)\left(\mathbb{C}^{n}\right)^{*}$. Then $\mathrm{SL}_{n+1} \times \mathrm{SL}_{n-1}$ acts on the $G$-invariants and the generators transform by the representations $\wedge^{n}\left(\mathbb{C}^{n+1}\right)$ (the determinants) and $\mathbb{C}^{n+1} \otimes \mathbb{C}^{n-1}$ (the contractions). By [We, Theorem 2.14.A], the relations are generated by those corresponding to

$$
\mathbb{C}^{n-1} \simeq \wedge^{n+1}\left(\mathbb{C}^{n+1}\right) \otimes \mathbb{C}^{n-1} \subset \wedge^{n}\left(\mathbb{C}^{n+1}\right) \otimes\left(\mathbb{C}^{n+1} \otimes \mathbb{C}^{n-1}\right) .
$$

We have a complete intersection since

$$
\left(\begin{array}{c}
n+1 \\
n
\end{array}\right)+n^{2}-1-(n-1)=n^{2}+1=\operatorname{dim} Z=\operatorname{dim} V-\operatorname{dim} G=2 n^{2}-\left(n^{2}-1\right) .
$$

Now we state a general theorem enabling us to show that $p_{m}^{*}: \mathcal{O}\left(Z_{m}\right) \rightarrow \mathcal{O}\left(V_{m}\right)^{G_{m}}$ is an isomorphism for all $m \geq 0$. Let $G$ be connected reductive and $V$ a $G$-module such that every semi-invariant is an invariant and such that $Z$ is a complete intersection. As before, let $Z^{\prime}$ denote the image of the points $V^{\prime}$ of $V$ where $d p$ has maximal rank. Let $Z_{m, 0}$ denote $Z_{m} \backslash Z_{m}^{\prime}$. Recall that $Z_{m}^{\prime}$ consists of smooth points of $Z_{m}$.

Theorem 4.3. Let $G$ and $V$ be as above. Let $(W, H)$ be a nontrivial slice representation and write $W=W^{H} \oplus W_{1}$ where $W_{1}$ is an H-module. Let $q: W_{1} \rightarrow Y:=W_{1} / / H$ be the quotient mapping and suppose that $\pi_{m}^{-1}(q(0)) \cap Y_{m, 0}$ has codimension at least 2 in $Y_{m}$ for all $m \geq 0$ and all $(W, H)$. Then $Z_{m}$ is normal for all $m$ and $p_{m}^{*}: \mathcal{O}\left(Z_{m}\right) \rightarrow \mathcal{O}\left(V_{m}\right)^{G_{m}}$ is an isomorphism for $m=0,1, \ldots, \infty$.

Proof. By Boutot [Bo], it is known that $Z$ has rational singularities. Then [Mu] shows that each $Z_{m}$ is irreducible, reduced and a complete intersection, and $Z_{\infty}$ is reduced and irreducible. We thus need only show that $Z_{m} \backslash Z_{m}^{\prime}$ has codimension at least two in $Z_{m}$ for all $m$. Let $S \subset Z$ be the stratum corresponding to $H$. Then Luna's slice theorem shows that a neighborhood of $S$ in $Z$ is locally isomorphic to $S \times Y$ (up to étale mappings). Thus $(S \times Y)_{m}^{\prime} \simeq S_{m} \times Y_{m}^{\prime}$. Now the points of $Y_{m, 0}$ either lie over the stratum $\{q(0)\}$ of $Y$ or they lie over a stratum $T \subset Y$ with a smaller isotropy group. By induction we can assume 
that $\pi_{m}^{-1}(T) \cap Y_{m, 0}$ has codimension at least two in $Y_{m}$. By hypothesis, $\pi_{m}^{-1}(q(0)) \cap Y_{m, 0}$ has codimension at least two in $Y_{m}$. Thus $Y_{m, 0}$ has codimension two in $Y_{m}$ and $Z_{m, 0}$ has codimension two in $Z_{m}$ over a neighborhood of $S$. Since this is true for all strata $S$, we find that $Z_{m, 0}$ has codimension two in $Z_{m}$, i.e., $Z_{m}$ is normal. Thus $p_{m}^{*}: \mathcal{O}\left(Z_{m}\right) \rightarrow \mathcal{O}\left(V_{m}\right)^{G_{m}}$ is an isomorphism for $m \leq \infty$.

Theorem 4.4. Let $(V, G)=\left((n+1) \mathbb{C}^{n}+(n-1)\left(\mathbb{C}^{n}\right)^{*}, \mathrm{SL}_{n}\right), n \geq 2$. Then $Z_{m}$ is normal for all $m \geq 0$ and $p_{m}^{*}: \mathcal{O}\left(Z_{m}\right) \rightarrow \mathcal{O}\left(V_{m}\right)^{G_{m}}$ is an isomorphism for $m=0,1, \ldots, \infty$.

Proof. Let us call our $n-1$ relations $f_{1}, \ldots, f_{n-1}$. Then the $f_{j}$ are bilinear, being linear in the determinants and the contractions. Let

$$
(\omega(t), \alpha(t))=\left(\sum_{i=1}^{m} t^{i} \omega_{i}, \sum_{i=1}^{m} t^{i} \alpha_{i}\right)
$$

be elements of $\pi_{m}^{-1}(p(0))$ where the $\omega_{i}$ correspond to the determinants and the $\alpha_{i}$ to the contractions. Then for $j=1, \ldots, n-1$ we have the equations $f_{j}(\omega(t), \alpha(t))=0 \bmod t^{m+1}$. Thus we get the equations

$$
\begin{aligned}
f_{j}\left(\omega_{1}, \alpha_{1}\right)=0, & f_{j}\left(\omega_{2}, \alpha_{1}\right)+f_{j}\left(\omega_{1}, \alpha_{2}\right)=0, \ldots \\
& \ldots, f_{j}\left(\omega_{m-1}, \alpha_{1}\right)+\cdots+f_{j}\left(\omega_{1}, \alpha_{m-1}\right)=0 .
\end{aligned}
$$

We have no conditions on $\omega_{m}$ and $\alpha_{m}$, and the equations above on the $\omega_{i}$ and $\alpha_{i^{\prime}}$ for $1 \leq i, i^{\prime} \leq m-1$ give something isomorphic to $Z_{m-2}$. Thus the dimension of $\pi_{m}^{-1}(p(0))$ is $\operatorname{dim} Z_{m-2}+\left(n^{2}+n\right)$ for $m \geq 2$ and the codimension of $\pi_{m}^{-1}(p(0))$ in $Z_{m}$ is

$$
\begin{aligned}
& \operatorname{dim} Z_{m}-\left(\operatorname{dim} Z_{m-2}\right)-\left(n^{2}+n\right) \\
& =(m+1-(m-1))\left(n^{2}+1\right)-\left(n^{2}+n\right)=n^{2}-n+2 \geq 4 .
\end{aligned}
$$

For $m=1$ we get the same codimension and for $m=0$ the codimension is $n^{2}+1 \geq 5$. Now we can use Lemma4.2 and Theorem 4.3 to finish the proof.

We consider later the case of $\left(n\left(\mathbb{C}^{n}+\left(\mathbb{C}^{n}\right)^{*}\right), \mathrm{SL}_{n}\right)$ whose quotient is a complete intersection.

Theorem 4.5. Let $(V, G)=\left(k \mathbb{C}^{2 n}, \mathrm{Sp}_{2 n}\right), n \geq 1$. Then $V$ is coregular for $k \leq 2 n+1, Z$ is a hypersurface for $k=2 n+2$ and is not a complete intersection for $k \geq 2 n+3$. When $k=2 n+2$, $Z_{m}$ is normal for all $m$ and $p_{m}^{*}: \mathcal{O}\left(Z_{m}\right) \rightarrow \mathcal{O}\left(V_{m}\right)^{G_{m}}$ is an isomorphism for $m=0,1, \ldots, \infty$.

Proof. The group $G$ preserves a canonical non-degenerate skew form on $\mathbb{C}^{2 n}$. If we use indices $1 \leq i<j \leq k$ for pairs of copies of $\mathbb{C}^{2 n}$, then the invariants of $V$ give a 2form $\omega=\sum \omega_{i j} e_{i} \wedge e_{j}$ and the relations of the $\omega_{i j}$ are given by the vanishing of $\omega^{n+1} \in$ $\wedge^{2 n+2}\left(\mathbb{C}^{k}\right) \otimes \mathbb{C}\left[\omega_{i j}\right]$. It follows that $V$ is coregular for $k \leq 2 n+1$ and has too many relations for $k \geq 2 n+3$. Thus we need only consider the hypersurface case $k=2 n+2$. The noncoregular slice representations of $V$ are of the same form as $V$ with $n$ replaced by a smaller $n^{\prime} \geq 1$, modulo trivial representations. We show that $Z_{m, 0}$ has codimension 4 in $Z_{m}$ which, by Theorem 4.3, establishes our result.

First consider the case where $m$ is at least $n+1$. Let

$$
\omega=\sum_{i=1}^{m} t^{i} \omega_{i} \in \pi_{m}^{-1}(p(0)) .
$$


Here the $\omega_{i}$ are elements of $\bigwedge^{2}\left(\mathbb{C}^{2 n+2}\right)_{i}$. Then we have the equations $\omega_{1}^{n+1}=0, \omega_{1}^{n} \wedge$ $\omega_{2}=0, \ldots$. The equations applied to $n+1$ of the $\omega_{i}$ such that the sum of the indices does not exceed $m$ gives a set isomorphic to $Z_{m-n-1}$, and there are no conditions on $\omega_{m-n+1}, \omega_{m-n+2}, \ldots, \omega_{m}$. Now $Z$ has dimension $\left(\begin{array}{c}2 n+2 \\ 2\end{array}\right)-1$ and it follows that the codimension of $\pi_{m}^{-1}(p(0))$ in $Z_{m}$ is

$$
\begin{aligned}
& ((m+1)-(m-n))\left(\left(\begin{array}{c}
2 n+2 \\
2
\end{array}\right)-1\right)-n\left(\begin{array}{c}
2 n+2 \\
2
\end{array}\right) \\
& =\left(\begin{array}{c}
2 n+2 \\
2
\end{array}\right)-n-1=2 n^{2}+2 n \geq 4 .
\end{aligned}
$$

For the cases $m=0, \ldots, n$ one gets that $\operatorname{codim} \pi_{m}^{-1}(p(0))=\operatorname{dim} Z-m$ which is even better. This completes the proof.

Theorem 4.6. Let $(V, G)=\left(k \mathbb{C}^{n}+\ell\left(\mathbb{C}^{n}\right)^{*}, \mathrm{GL}_{n}\right), n \geq 1, k \geq \ell$. Then $V$ is coregular if $\ell \leq n$, there are too many relations if $\ell \geq n+1$ and $k+\ell \geq 2 n+3$ and $Z$ is a hypersurface if $k=\ell=n+1$. In this last case we have that $Z_{m}$ is normal for all $m$ and $p_{m}^{*}: \mathcal{O}\left(Z_{m}\right) \rightarrow \mathcal{O}\left(V_{m}\right)^{G_{m}}$ is an isomorphism for $m=0,1, \ldots, \infty$.

Proof. The invariants are just the contractions $\alpha_{i j}, 1 \leq i \leq k, 1 \leq j \leq \ell$ and the relations are $\operatorname{det}_{r, s=1}^{n+1} \alpha_{i_{r}, j_{s}}=0$ where the $i_{r}$ are distinct and the $j_{s}$ are distinct. This shows that $V$ is coregular for $\ell \leq n$ and that $Z$ is not a complete intersection for $\ell \geq n+2$ or $\ell=n+1$ and $k>n+1$. We now consider the case $k=\ell=n+1$. Then all the nontrivial slice representations have the same form, with $n$ replaced by a smaller $n^{\prime} \geq 1$. The codimension of $p(0)$ in $Z_{0}$ is $\operatorname{dim} Z=(n+1)^{2}-1 \geq 3$. For $m \geq n+1$ one can use the techniques as above to show that the codimension of $\pi_{m}^{-1}(p(0))$ in $Z_{m}$ is at least

$$
\begin{aligned}
& \operatorname{dim} Z_{m}-\operatorname{dim} Z_{m-n-1}-n(n+1)^{2} \\
& =((m+1)-(m-n))\left((n+1)^{2}-1\right)-n(n+1)^{2}=n(n+1) \geq 2 .
\end{aligned}
$$

For $1 \leq m \leq n$ the codimension is at least as great. Thus, as before, we find that $Z_{m}$ is normal for all $m$ and the theorem follows.

Theorem 4.7. Let $(V, G)=\left(k \mathbb{C}^{n}, \mathrm{SO}_{n}\right), n \geq 2$. Then $V$ is coregular for $k<n$ and has too many relations if $k>n$. If $k=n$, then $Z$ is a hypersurface and $Z_{m}$ is normal for all $m$. Hence $p_{m}^{*}: \mathcal{O}\left(Z_{m}\right) \rightarrow \mathcal{O}\left(V_{m}\right)^{G_{m}}$ is an isomorphism for $m=0,1, \ldots, \infty$.

Proof. We leave it to the reader to show that there are too many relations if $k>n$ and that $V$ is coregular for $k<n$. Consider the case that $k=n$. The invariants of $V$ are generated by the inner products $\alpha_{i j}, 1 \leq i, j \leq n$ and the determinant $d$. The relation is $\operatorname{det}_{i, j=1}^{n} \alpha_{i j}=d^{2}$. The nontrivial slice representations of $V$ are just those for $n$ replaced by $n^{\prime}$ where $2 \leq n^{\prime}<n$, up to trivial factors, so it is enough to show that $Y_{m}:=\pi_{m}^{-1}(p(0))$ has codimension at least 2 in $Z_{m}$ for $m \geq 0$. Let $\alpha(t)=\sum_{i=1}^{m} t^{i} \alpha_{i}$ and $d(t)=\sum_{i=1}^{m} t^{i} d_{i}$ be elements of $Y_{m}$ where $m \geq n$. For now suppose that $n=2 \ell$ is even. Then the equations $\operatorname{det}(\alpha(t))=d(t)^{2}$ force $d_{1}=\cdots=d_{\ell-1}=0$. The $n$th equation is $\operatorname{det}\left(\alpha_{1}\right)=d_{\ell}^{2}$ and one can see that the dimension of $Y_{m}$ is $\operatorname{dim} Z_{m-n}+(n-1)(\operatorname{dim} Z+1)-(n-2) / 2$ so that the codimension of $Y_{m}$ in $Z_{m}$ is

$$
\operatorname{dim} Z-(n-1)+(n-2) / 2=\frac{1}{2} n(n+1)-n+1+\frac{1}{2}(n-2)=\frac{1}{2} n^{2}
$$


which is at least 2. If $m<n$, then we get the estimate that $\operatorname{codim} Y_{m} \leq \operatorname{dim} Z-m+\left[\frac{m}{2}\right]$ which is even better.

Now we consider the case that $n=2 \ell+1$ is odd. Then looking at the coefficient of $t^{n}$ we get the equation $\operatorname{det}\left(\alpha_{1}\right)=0$ since the equations with lower powers of $t$ force $d_{1}=\cdots=$ $d_{\ell}=0$. The coefficient of $t^{n+1}$ gives an inhomogeneous equation for $\alpha_{1}$ and $\alpha_{2}$ with right hand side $d_{\ell+1}^{2}$. But the solution space of the inhomogeneous equation has dimension at most that of the homogeneous equation. Hence we can estimate the dimension of $Y_{m}$ by replacing the inhomogeneous equations by the homogeneous equations. Thus we get the estimate $\operatorname{dim} Y_{m}=\operatorname{dim} Z_{m-n}+(n-1)(\operatorname{dim} Z+1)-(n-1) / 2$ and for the codimension we get

$$
\operatorname{dim} Z-(n-1)+\frac{n-1}{2}=\frac{1}{2} n(n+1)-n+1+\frac{1}{2}(n-1)=\frac{1}{2} n^{2}+\frac{1}{2} .
$$

As before, the estimates for $0 \leq m<n$ are even better, so $Y_{m}$ has codimension 2 and $Z_{m}$ is normal.

There is one case left, which needs no new techniques.

Theorem 4.8. Let $(V, G)=\left(n \mathbb{C}^{n}+n\left(\mathbb{C}^{n}\right)^{*}, \mathrm{SL}_{n}\right), n \geq 2$. Then $Z_{m}$ is normal and $p_{m}^{*}: \mathcal{O}\left(Z_{m}\right) \rightarrow$ $\mathcal{O}\left(V_{m}\right)^{G_{m}}$ is an isomorphism for $m=0,1, \ldots, \infty$.

Proof. The generators of $\mathcal{O}(V)^{G}$ are the contractions $\alpha_{i j}$ and the determinants $d$ and $e$ of the $n$ copies of $\mathbb{C}^{n}$ and its dual. The relation is $\operatorname{det}\left(\alpha_{i j}\right)=d e$. The nontrivial slice representations, up to trivial factors, have the same form, with $n$ replaced by a smaller $n^{\prime} \geq 2$. As above, one can show that $\pi_{m}^{-1}(p(0))$ always has codimension at least 4 in $Z_{m}$ which gives the result.

\section{REPRESENTATIONS OF $\mathbb{C}^{*}$}

The main theorem of this section is the following.

Theorem 5.1. Let $G=\mathbb{C}^{*}$ and let $V$ be a $G$-module all of whose weights are \pm 1 . Then $p_{\infty}^{*}: \mathcal{O}\left(Z_{\infty}\right) \rightarrow$ $\mathcal{O}\left(V_{\infty}\right)^{G_{\infty}}$ is an isomorphism and $\mathcal{O}\left(Z_{\infty}\right)$ is integrally closed.

Corollary 5.2. Let $V$ be a $G=\mathbb{C}^{*}$ module with nonzero positive weights $r_{1} \leq \cdots \leq r_{d}, d \geq 1$, and nonzero negative weights $-s_{1} \leq \cdots \leq-s_{e}, e \geq 1$, where we may assume that the greatest common divisor of the $r_{i}$ and $s_{j}$ is one. Then $p_{\infty}^{*}$ is surjective only in the following cases.

(1) $d=1$ and $r_{1}=1$ or $e=1$ and $s_{1}=1$. Here $Z^{\prime}=Z$ so that $p_{m}^{*}$ is an isomorphism for all $m$.

(2) All the $r_{i}$ and $s_{j}$ are 1 .

Proof. Suppose that there are weights $r_{i}$ and $-s_{j}$ neither of which is 1 . Let $W$ be the corresponding two dimensional submodule of $V$. Then the null cone of $W$ consists of two non-reduced hypersurfaces, hence $W$ is not good and neither is $V$. Thus, without loss of generality, we can assume that $r_{i}=1$ for all $i$. If $d=1$, then we are in case (1) and one easily sees that $Z^{\prime}=Z$. Suppose that $d>1$. If $s_{j}=s \neq 1$ for some $j$, then $V$ contains a submodule $W$ with weights 1,1 and $-s$. Let $x_{1}, x_{2}$ and $y$ be the corresponding coordinate functions. The generators of the invariants have degree $s+1$. Now $\left(x_{1} D x_{2}-x_{2} D x_{1}\right) x_{1}^{s-2} y$ is an invariant of $G_{\infty}$ of weight one and degree $s+1$ but is not $D$ applied to a generator of $\mathcal{O}(W)^{\mathbb{C}^{*}}$. Hence $W$ is not good. Thus we have to be in case (2). 
We now prove the theorem. We may assume that $V^{G}=(0)$ and that the weights 1 and -1 have multiplicity $n$. Let $x_{1}, \ldots, x_{n}$ be coordinate functions corresponding to the positive weights and let $y_{1}, \ldots, y_{n}$ be coordinate functions corresponding to the negative weights. Set $x_{i}^{(k)}:=D^{k} x_{i}$ and $y_{j}^{(k)}=D^{k} y_{j}$ for $k \geq 0$. Then the $x_{i}^{(k)}$ and $y_{j}^{(k)}$ are coordinate functions on $V_{\infty}$. We order the $x_{i}^{(k)}$ so that $x_{i}^{(k)}<x_{j}^{(\ell)}$ if $k<\ell$ or $k=\ell$ and $i<j$. We similarly order the $y_{i}^{(k)}$. The $G$-invariants of $R:=\mathcal{O}\left(V_{\infty}\right)$ are linear combinations of monomials $W:=u_{1} \cdots u_{r} v_{1} \cdots v_{r}$ where each $u_{i}$ is an $x_{j}^{(k)}$ and each $v_{i}$ is some $y_{j^{\prime}}^{\left(k^{\prime}\right)}$ and we have that $u_{1} \leq \cdots \leq u_{r}$ and $v_{1} \leq \cdots \leq v_{r}$. We say that $W$ is a word in standard form. We define the weight wt $(W)$ of $W$ to be the sums of the exponents of the $x_{i}^{(k)}$ and $y_{j}^{(\ell)}$ occurring in $W$. Let $W^{\prime}=u_{1}^{\prime} \cdots u_{r}^{\prime} v_{1}^{\prime} \cdots v_{r}^{\prime}$ be another monomial in standard form. We say that $W<W^{\prime}$ if

(1) $\operatorname{wt}(W)<\operatorname{wt}\left(W^{\prime}\right)$,

(2) $\operatorname{wt}(W)=\operatorname{wt}\left(W^{\prime}\right), u_{1} \cdots u_{s} v_{1} \cdots v_{s}=u_{1}^{\prime} \cdots u_{s}^{\prime} v_{1}^{\prime} \cdots v_{s}^{\prime}$ and $u_{s+1}<u_{s+1}^{\prime}$ where $0 \leq s<$ $r$, or

(3) $\operatorname{wt}(W)=\operatorname{wt}\left(W^{\prime}\right), u_{1} \cdots u_{s+1} v_{1} \cdots v_{s}=u_{1}^{\prime} \cdots u_{s+1}^{\prime} v_{1}^{\prime} \cdots v_{s}^{\prime}$ and $v_{s+1}$

$<v_{s+1}^{\prime}$ where $0 \leq s<r$.

We say that $W$ is admissible if it is in standard form, $v_{1}$ is some $y_{j}$ and for $1 \leq s<r$, $D\left(v_{s}\right)>v_{s+1}$. Given $0 \neq h \in \mathcal{O}\left(V_{\infty}\right)^{G_{\infty}}$ of degree $2 r$ and weight $m$ it is a sum $\sum_{i} c_{i} W_{i}$ where the $W_{i}$ are distinct and in standard form, the $c_{i}$ are nonzero scalars and $\operatorname{wt}\left(W_{i}\right)=m$ and $\operatorname{deg}\left(W_{i}\right)=2 r$ for all $i$. We define the leading term $M(h)$ to be the greatest $W_{i}$. We will eventually show that $M(h)$ is admissible.

The ring $\mathcal{O}(Z)$ has generators $f_{i j}$ (corresponding to $x_{i} y_{j}$ ) and relations $R_{a b, c d}=f_{a b} f_{c d}-$ $f_{a d} f_{c b}, 1 \leq a, b, c, d \leq n$ and $a \neq c, b \neq d$. Then $\mathcal{O}\left(Z_{\infty}\right)$ has generators $f_{i j}^{(k)}:=D^{k} f_{i j}$ and relations generated by the $D^{k} R_{a b, c d}$. Of course, $p_{\infty}^{*} f_{i j}^{(k)}=D^{k}\left(x_{i} y_{j}\right)$. We define a partial order on the $f_{i j}^{(k)}$ where $f_{i j}^{(k)} \leq f_{i^{\prime} j^{\prime}}^{\left(k^{\prime}\right)}$ if

(1) $k+2 \leq k^{\prime}$,

(2) $k+1=k^{\prime}$ and $i \leq i^{\prime}$ or $j \leq j^{\prime}$, or

(3) $k=k^{\prime}$ and $i \leq i^{\prime}$ and $j \leq j^{\prime}$.

We say that a monomial $f_{i_{1}, j_{1}}^{\left(k_{1}\right)} \cdots f_{i_{r}, j_{r}}^{\left(k_{r}\right)}$ is standard if $f_{i_{1}, j_{1}}^{\left(k_{1}\right)} \leq \cdots \leq f_{i_{r}, j_{r}}^{\left(k_{r}\right)}$.

Lemma 5.3. The algebra $\mathcal{O}\left(Z_{\infty}\right)$ is spanned by the standard monomials.

Proof. Suppose that $f_{a b}^{(k)} f_{c d}^{(\ell)}$ and $f_{c d}^{(\ell)} f_{a b}^{(k)}$ are not standard. First suppose that $k=\ell$. We may assume that $a>c$ and $b<d$. Consider the relation $D^{2 k}\left(f_{a b} f_{c d}-f_{a d} f_{c b}\right)=0$. It is a sum $\sigma+f_{a b}^{(k)} f_{c d}^{(k)}-f_{a d}^{(k)} f_{c b}^{(k)}$ where the terms in $\sigma$ are standard and involve an $f$ with weight higher than $k$. We replace $f_{a b}^{(k)} f_{c d}^{(k)}$ by $f_{a d}^{(k)} f_{c b}^{(k)}-\sigma$. The new expression is a sum of standard terms and is "larger" than $f_{a b}^{(k)} f_{c d}^{(k)}$ in that each term has a factor with a higher weight or contains the term $f_{a d}^{(k)}$ which is larger than $f_{a b}^{(k)}$ and $f_{c d}^{(k)}$ in our partial order.

Now suppose that $\ell=k+1$ and our terms are not standard. Then $a>c$ and $b>d$. Consider the relation $D^{2 k+1}\left(f_{a b} f_{c d}-f_{a d} f_{c b}\right)=0$. Expanding we get a sum

$$
\sigma+f_{a b}^{(k)} f_{c d}^{(k+1)}+f_{a b}^{(k+1)} f_{c d}^{(k)}-f_{a d}^{(k)} f_{c b}^{(k+1)}-f_{a d}^{(k+1)} f_{c b}^{(k)}
$$


where $\sigma$ is a sum of standard terms with a factor of weight at least $k+2$. Now the term $f_{a b}^{(k+1)} f_{c d}^{(k)}$ is standard and the factor $f_{a b}^{(k+1)}$ is larger than $f_{a b}^{(k)}$ and $f_{c d}^{(k+1)}$ in our partial order. Similarly, $f_{a d}^{(k)} f_{c b}^{(k+1)}$ is standard with $f_{c b}^{(k+1)}$ larger than $f_{a b}^{(k)}$ and $f_{c d}^{(k+1)}$. Similarly for $f_{a d}^{(k+1)} f_{c b}^{(k)}$. Hence we can replace $f_{a b}^{(k)} f_{c d}^{(k+1)}$ by larger standard terms.

Now it follows by induction that any monomial in the $f_{i j}^{(k)}$ is a sum of standard monomials.

Let $w=f_{i_{1}, j_{1}}^{\left(k_{1}\right)} \cdots f_{i_{r}, j_{r}}^{\left(k_{r}\right)}$ be standard. We construct a word

$$
L(w)=u_{1} \cdots u_{r} v_{1} \cdots v_{r}
$$

such that

(1) $u_{1} \leq \cdots \leq u_{r}$ and $v_{1} \leq \cdots \leq v_{r}$, i.e., $L(w)$ is a standard word.

(2) $u_{s} v_{s}$ is a monomial occurring in $p_{\infty}^{*}\left(f_{i_{s}, j_{s}}^{\left(k_{s}\right)}\right), 1 \leq s \leq r$.

(3) $v_{1}$ is a $y_{i}$ and $D v_{s}>v_{s+1}, 1 \leq s<r$, i.e., $L(w)$ is admissible.

Clearly we must have that $u_{1} v_{1}=x_{i_{1}}^{\left(k_{1}\right)} y_{j_{1}}^{(0)}$. Suppose that we have determined $u_{1}, \ldots, u_{r-1}$ and $v_{1}, \ldots, v_{r-1}$ satisfying (1)-(3) for $r$ replaced by $r-1$. We have that $u_{r-1}=x_{i_{r-1}}^{(a)}$ and $v_{r-1}=y_{j_{r-1}}^{(b)}$ where $a+b=k_{r-1}$. Suppose that $k_{r}=k_{r-1}$. Then $i_{r-1} \leq i_{r}$ and $j_{r-1} \leq j_{r}$ so $u_{r}=x_{i_{r}}^{(a)}$ and $v_{r}=y_{j_{r}}^{(b)}$ satisfy our conditions. If $k_{r}=k_{r-1}+1$ and $i_{r-1}>i_{r}$, then $j_{r-1} \leq j_{r}$ and we set $u_{r}=x_{i_{r}}^{(a+1)}$ and $v_{r}=y_{j_{r}}^{(b)}$. The case where $j_{r-1}>j_{r}$ is similar. If $i_{r-1} \leq i_{r}$ and $j_{r-1} \leq j_{r}$, then we set $u_{r}=x_{i_{r}}^{(a+1)}, v_{r}=y_{j_{r}}^{(b)}$. The case $k_{r} \geq 2+k_{r-1}$ is handled similarly.

Proposition 5.4. Let $w$ be as above. Then $L(w)=M\left(p_{\infty}^{*}(w)\right)$.

Proof. We have that $L(w)=u_{1} \cdots u_{r} v_{1} \cdots v_{r}$ where $u_{s}=x_{i_{s}}^{\left(a_{s}\right)}$ and $v_{s}=y_{j_{s}}^{\left(b_{s}\right)}$ and $a_{s}+b_{s}=k_{s}$, $1 \leq s \leq r$. Now $M:=M\left(p_{\infty}^{*}(w)\right)=u_{1}^{\prime} \cdots u_{r}^{\prime} v_{1}^{\prime} \cdots v_{r}^{\prime}$ where each $u_{s}^{\prime}$ is some $x_{i_{s}^{\prime}}^{a_{s}^{\prime}}$ and each $v_{s}^{\prime}$ is some $y_{j_{s}^{\prime}}^{b_{s}^{\prime}}$.

First we prove that $u_{1}^{\prime}=u_{1}$ and that $v_{1}^{\prime}=v_{1}$ where $u_{1}=x_{i_{1}}^{\left(k_{1}\right)}$ and $v_{1}=y_{j_{1}}^{(0)}$. Since $f_{i_{1}, j_{1}}^{\left(k_{1}\right)}$ is a factor of $w$, some $u_{s}^{\prime}$ is $x_{i_{1}}^{a}$ where $a \leq k_{1}$. Hence $u_{1}^{\prime} \leq u_{1}$ and by maximality of $M$ we must have equality. It follows that the monomial of $p_{\infty}^{*}\left(f_{i_{1}, j_{1}}^{\left(k_{1}\right)}\right)$ which occurs in $M$ is $x_{i_{1}}^{\left(k_{1}\right)} y_{j_{1}}^{(0)}$. Hence some $v_{s}^{\prime}$ is $y_{j_{1}}^{(0)}$ and $v_{1}^{\prime} \leq y_{j_{1}}^{(0)}$. By maximality of $M$ we have $v_{1}^{\prime}=v_{1}$.

Now suppose by induction that $u_{i}=u_{i}^{\prime}$ and $v_{i}=v_{i}^{\prime}$ for $i<r$. Then $u_{r}^{\prime} v_{r}^{\prime}$ is a monomial $x_{i_{r}}^{\left(a_{r}^{\prime}\right)} y_{j_{r}}^{\left(b_{r}^{\prime}\right)}$ occurring in $p_{\infty}^{*}\left(f_{i_{r}, j_{r}}^{\left(k_{r}\right)}\right)$ where $a_{r}^{\prime}+b_{r}^{\prime}=k_{r}$. If $j_{r-1} \leq j_{r}$, then the largest possible $a_{r}^{\prime}$ is $a_{r}=k_{r}-b_{r-1}$ and we have that $M=L(w)$. If $j_{r-1}>j_{r}$, one has $a_{r}^{\prime}=a_{r}=k_{r}-b_{r-1}-1$ and again $M=L(w)$. Hence we always have $L(w)=M\left(p_{\infty}^{*}(w)\right)$.

If $W$ is admissible, then it is clear that there is a unique $w$ with $W=L(w)$, hence we have the following

Lemma 5.5. The function $w \mapsto L(w)$ is a bijective mapping from standard monomials to admissible monomials. Hence the standard monomials are linearly independent.

Since the smallest possible $u_{1} v_{1}$ is $f_{11}=x_{1} y_{1}$, multiplication by $f_{11}$ is injective on linear combinations of standard monomials. Hence $f_{11}$ is not a zero divisor in $\mathcal{O}\left(Z_{\infty}\right)$ and 
the mapping from $\mathcal{O}\left(Z_{\infty}\right)$ to its localization $\mathcal{O}\left(Z_{\infty}\right)_{f_{11}}$ is injective. By Corollary 3.7 this localization is isomorphic to $\left(\mathcal{O}\left(V_{\infty}\right)^{G_{\infty}}\right)_{\left(x_{1} y_{1}\right)}$. Hence we have

Proposition 5.6. Let $G=\mathbb{C}^{*}$ and let $V$ be a $G$-module with weights \pm 1 of multiplicity $n$, as above. The mapping $p_{\infty}^{*}: \mathcal{O}\left(Z_{\infty}\right) \rightarrow \mathcal{O}\left(V_{\infty}\right)^{G_{\infty}}$ is injective, hence $\mathcal{O}\left(Z_{\infty}\right)$ is reduced. Given $h \in \mathcal{O}\left(V_{\infty}\right)^{G_{\infty}}$ there is an $s \geq 0$ and an $f \in \mathcal{O}\left(Z_{\infty}\right)$ such that $\left(x_{1} y_{1}\right)^{s} h=p_{\infty}^{*}(f)$.

Corollary 5.7. Let $h \in \mathcal{O}\left(V_{\infty}\right)^{G_{\infty}}$. Then $M(h)$ is admissible.

Proof. There is an $s \geq 0$ such that $\left(x_{1} y_{1}\right)^{s} h$ is in the image of $\mathcal{O}\left(Z_{\infty}\right)$. Hence $M\left(\left(x_{1} y_{1}\right)^{s} h\right)$ is admissible. But $M\left(\left(x_{1} y_{1}\right)^{s} h\right)=\left(x_{1} y_{1}\right)^{s} M(h)$ and hence $M(h)$ is admissible.

Proof of Theorem 5.1 Let $h \in \mathcal{O}\left(V_{\infty}\right)^{G_{\infty}}$ have a fixed degree and weight. Let $W=M(h)$. Then, as we saw before, there is a canonical standard monomial $w$ such that $M\left(p_{\infty}^{*}(w)\right)=$ $W$. Then for some constant $c, M\left(h-p_{\infty}^{*}(c w)\right)<M(h)$. By induction, then, we get that $h \in p_{\infty}^{*} \mathcal{O}\left(Z_{\infty}\right)$. Since the group $G_{\infty}$ is connected, one shows as usual that $\mathcal{O}\left(V_{\infty}\right)^{G_{\infty}}$ is integrally closed, hence so is $\mathcal{O}\left(Z_{\infty}\right)$.

\section{SOME REPRESENTATIONS OF $\mathrm{SL}_{n}$}

In this section we consider the case where $(V, G)=\left(\ell \mathbb{C}^{n}, \mathrm{SL}_{n}\right)$. We use a version of standard monomial theory to prove that $p_{\infty}^{*} \mathcal{O}\left(Z_{\infty}\right)=\mathcal{O}\left(V_{\infty}\right)^{G_{\infty}}$. For $n=2$ one can show that $p_{\infty}^{*}$ is injective, but for $n \geq 3$ this fails, as we show in Example 6.6.

Consider pairs $(j, k)$ where $1 \leq j \leq \ell$ and $k \geq 0$. For each $j$ let $\left\{x_{i}^{(j, 0)}\right\}_{i=1}^{n}$ denote the usual coordinate functions on the $j$ th copy of $\mathbb{C}^{n}$. Let $x_{i}^{(j, k)}$ denote $(1 / k !) D^{k} x_{i}^{(j, 0)}$. Then the $x_{i}^{(j, k)}$ are coordinate functions on $V_{\infty}$. Let $\omega=(j, k)$. Then we write $\omega<\omega^{\prime}=\left(j^{\prime}, k^{\prime}\right)$ if $k<k^{\prime}$ or $k=k^{\prime}$ and $j<j^{\prime}$. We write $x_{i}^{\omega}<x_{i^{\prime}}^{\omega^{\prime}}$ if $\omega<\omega^{\prime}$ or $\omega=\omega^{\prime}$ and $i^{\prime}<i$. Thus we have $x_{1}^{\omega}>\cdots>x_{n}^{\omega}$ for any $\omega$. We inductively define a monomial order as follows. Let $M=m x_{i}^{\omega}$ (resp. $\left.M^{\prime}=m^{\prime} x_{i^{\prime}}^{\omega^{\prime}}\right)$ be a monomial where $x_{i}^{\omega}$ (resp. $\left.x_{i^{\prime}}^{\omega^{\prime}}\right)$ is the largest variable occurring in $M$ (resp. $M^{\prime}$ ). If $\operatorname{deg}(M)<\operatorname{deg}\left(M^{\prime}\right)$ or $\operatorname{deg} M=\operatorname{deg}\left(M^{\prime}\right)$ and $\operatorname{wt}(M)<\operatorname{wt}\left(M^{\prime}\right)$, then $M<M^{\prime}$. If $M$ and $M^{\prime}$ have the same degree and weight, then $M<M^{\prime}$ if $x_{i}^{\omega}<x_{i^{\prime}}^{\omega^{\prime}}$ or $x_{i}^{\omega}=x_{i^{\prime}}^{\omega^{\prime}}$ and $m<m^{\prime}$. If $h \neq 0$ is in $\mathcal{O}\left(V_{\infty}\right)^{G_{\infty}}$, we let $L(h)$ denote the lowest monomial occurring with nonzero coefficient in the expression of $h$ in the $x_{i}^{\omega}$.

Let $x^{\omega}$ denote the vector $\left(x_{1}^{\omega}, \ldots, x_{n}^{\omega}\right)$. By classical invariant theory, the algebra $\mathcal{O}\left(V_{\infty}\right)^{G}$ is generated by determinants $\left[x^{\omega_{1}}, \ldots, x^{\omega_{n}}\right]$.

Remarks 6.1. (1) We have $L\left(\left[x^{\omega_{1}}, \ldots, x^{\omega_{n}}\right]\right)=x_{1}^{\omega_{1}} \cdots x_{n}^{\omega_{n}}$ where $\omega_{1}<\cdots<\omega_{n}$.

(2) Let $1 \leq j_{1}<\cdots<j_{n} \leq \ell$ and let $k=n a+b$ where $a, k \in \mathbb{Z}^{+}$and $0 \leq b<n$. Then

$$
\begin{aligned}
& L\left(D^{k}\left(\left[x^{\left(j_{1}, 0\right)}, \ldots, x^{\left(j_{n}, 0\right)}\right]\right)\right) \\
& =L\left(\left[x^{\left(j_{b+1}, a\right)}, \ldots, x^{\left(j_{n}, a\right)}, x^{\left(j_{1}, a+1\right)}, \ldots, x^{\left(j_{b}, a+1\right)}\right]\right) .
\end{aligned}
$$

Let

$$
W=\left(\begin{array}{cccc}
\omega_{1,1} & \omega_{1,2} & \ldots & \omega_{1, n} \\
\vdots & \vdots & \omega_{i, j} & \vdots \\
\omega_{s, 1} & \omega_{s, 2} & \cdots & \omega_{s, n}
\end{array}\right)
$$

where the $\omega_{i, j}$ are pairs as above. We call $W$ a tableau and we say that $W$ is standard if the rows are strictly increasing and the columns are nondecreasing. To each row $\omega_{r, 1}, \ldots, \omega_{r, n}$ 
of $W$ we associate the determinant $\left[x^{\omega_{r, 1}}, \ldots, x^{\omega_{r, n}}\right]$ and to $W$ we associate the product $P(W)$ of the determinants determined by the rows. Let $Q(W)$ denote $\prod_{s} \prod_{t=1}^{n} x_{t}^{\omega_{s, t}}$.

Proposition 6.2. $\quad$ (1) The $P(W)$ for $W$ a standard tableau are a basis of $\mathcal{O}\left(V_{\infty}\right)^{G}$.

(2) The mapping $W \mapsto Q(W)$ is injective and $Q(W)=L(P(W)$ ).

(3) If $0 \neq h \in \mathcal{O}\left(V_{\infty}\right)^{G}$, then $L(h)=Q(W)$ for a standard $W$.

Proof. Part (1) is just standard monomial theory and (2) is obvious. Let $h$ be as in (3). Then $h$ is a sum $\sum_{i} c_{i} P\left(W_{i}\right)$ where the $c_{i}$ are nonzero and the $W_{i}$ are standard and distinct. Then the $Q\left(W_{i}\right)$ are distinct and $L(h)$ is the minimum of the $Q\left(W_{i}\right)$.

For $\omega=(j, k)$ with $\operatorname{wt}(\omega)=k>0$, let $\tilde{\omega}$ denote $(j, k-1)$. Let $X \in \mathfrak{g}=\mathfrak{s l}_{n}$ be the element which sends $x_{n} \rightarrow x_{1}$ and annihilates $x_{i}$ for $i<n$. Here the $x_{i}$ are the usual coordinate functions on $\mathbb{C}^{n}$. Let $F$ denote $t X \in \mathfrak{g}[t]$. Then $F$ and $\mathfrak{g}$ generate $\mathfrak{g}[t]$ so that $\mathcal{O}\left(V_{\infty}\right)^{G_{\infty}}=\mathcal{O}\left(V_{\infty}\right)^{\mathfrak{g}[t]}=\mathcal{O}\left(V_{\infty}\right)^{G} \cap \mathcal{O}\left(V_{\infty}\right)^{F}$. Now $F$ annihilates the $x_{n}^{(j, 0)}$ and the $x_{i}^{\omega}$ for $i \neq n$, and it sends $x_{n}^{\omega}$ to $x_{1}^{\tilde{\omega}}$ when $\operatorname{wt}(\omega)>0$. For wt $(\omega)>0$ let $y_{2}^{\omega}$ denote $x_{n}^{\omega}$ and let $y_{1}^{\omega}$ denote $x_{1}^{\tilde{\omega}}$. Then the action of $F$ on the $y_{i}^{\omega}$ is the standard action of the Lie algebra of the maximal unipotent subgroup $U$ of $\mathrm{SL}_{2}$. Consider the symmetric algebra $A$ in the $y_{i}^{\omega}$ for $\operatorname{wt}(\omega)>0$. For fixed $\omega$, the invariants of $U$ acting on the subalgebra generated by $y_{1}^{\omega}$ and $y_{2}^{\omega}$ are generated by $y_{1}^{\omega}$. Then it follows from [We, Theorem 2.5.A] that the $U$-invariants of $A$ are generated by the $y_{1}^{\omega}$ and the determinants $I_{\omega^{\prime}}^{\omega}:=\left[y^{\omega}, y^{\omega^{\prime}}\right]=y_{1}^{\omega} y_{2}^{\omega^{\prime}}-y_{1}^{\omega^{\prime}} y_{2}^{\omega}$. Then we have the following relations:

$$
I_{\omega_{2}}^{\omega_{1}}=-I_{\omega_{1}}^{\omega_{2}}, \quad I_{\omega_{2}}^{\omega_{1}} I_{\omega_{4}}^{\omega_{3}}=I_{\omega_{2}}^{\omega_{3}} I_{\omega_{4}}^{\omega_{1}}+I_{\omega_{3}}^{\omega_{1}} I_{\omega_{4}}^{\omega_{2}}, \quad I_{\omega_{2}}^{\omega_{1}} y_{1}^{\omega_{3}}=I_{\omega_{2}}^{\omega_{3}} y_{1}^{\omega_{1}}+I_{\omega_{3}}^{\omega_{1}} y_{1}^{\omega_{2}}
$$

where $\operatorname{wt}\left(\omega_{i}\right)>0, i=1, \ldots, 4$. Let

$$
Y=\left(\begin{array}{cc}
\omega_{1} & \omega_{1}^{\prime} \\
\vdots & \vdots \\
\omega_{q} & \omega_{q}^{\prime} \\
\omega_{q+1} & \\
\vdots & \\
\omega_{q+r} &
\end{array}\right)
$$

be an array of our pairs $(j, k)$ (a tableau) where $k>0$. As usual, we say that $Y$ is standard if the pairs are strictly increasing in the rows and nondecreasing as one goes down the columns. Let $\widetilde{P}(Y)$ denote the polynomial $\left[y^{\omega_{1}}, y^{\omega_{1}^{\prime}}\right] \cdots\left[y^{\omega_{q}}, y^{\omega_{q}^{\prime}}\right] y_{1}^{\omega_{q+1}} \cdots y_{1}^{\omega_{q+r}}$. We say that $\widetilde{P}(Y)$ is a standard monomial. Then the relations above show that $A^{F}=A^{U}$ has basis the standard monomials.

We have the induced monomial order on $A \subset \mathcal{O}\left(V_{\infty}\right)$. Then for $\omega<\omega^{\prime}$ we have

$$
L\left(\left[y^{\omega}, y^{\omega^{\prime}}\right]\right)=y_{1}^{\omega^{\prime}} y_{2}^{\omega}=x_{1}^{\tilde{\omega}^{\prime}} x_{n}^{\omega} .
$$

Lemma 6.3. Let $Y$ be as above. Then the leading term of $L(\widetilde{P}(Y))$ is

$$
y_{1}^{\omega_{1}^{\prime}} y_{2}^{\omega_{1}} \cdots y_{1}^{\omega_{q}^{\prime}} y_{2}^{\omega_{q}} y_{1}^{\omega_{q+1}} \cdots y_{1}^{\omega_{q+r}}=x_{1}^{\tilde{\omega}_{1}^{\prime}} x_{n}^{\omega_{1}} \cdots x_{1}^{\tilde{\omega}_{q}^{\prime}} x_{n}^{\omega_{q}} x_{1}^{\omega_{q+1}} \cdots x_{1}^{\omega_{q+r}} .
$$

Let $A^{\prime}$ be the subalgebra of $\mathcal{O}\left(V_{\infty}\right)$ generated by $A$ and the $x_{n}^{(j, 0)}, 1 \leq j \leq \ell$, and let $B$ be the subalgebra of $\mathcal{O}\left(V_{\infty}\right)$ generated by the $x_{i}^{\omega}$ for $1<i<n$. Then $\mathcal{O}\left(V_{\infty}\right)$ is the tensor product $A^{\prime} \otimes_{\mathbb{C}} B$. 
Corollary 6.4. (1) Let $h \in\left(A^{\prime}\right)^{F}$. Then $L(h)$ is of the form $f L(\widetilde{P}(Y))$ where $Y$ is standard and $f$ is a homogeneous polynomial in the $x_{n}^{(j, 0)}$.

(2) Let $h \in \mathcal{O}\left(V_{\infty}\right)^{F}$. Then $L(h)$ is of the from $f L(\widetilde{P}(Y))$ where $Y$ is standard and $f$ is a homogeneous polynomial in the $x_{n}^{(j, 0)}$ and $x_{t}^{\omega}$ for $1<t<n$.

(3) Let $h \in \mathcal{O}\left(V_{\infty}\right)^{G_{\infty}}$. Then $L(h)=L(P(W))$ where $W=\left(\omega_{i j}\right)$ is as in (6.1). For any $i$ with $1 \leq i \leq s$, if $\operatorname{wt}\left(\omega_{i, n}\right)>0$, then $\tilde{\omega}_{i, n}<\omega_{i, 1}$.

Proof. Parts (1) and (2) are immediate. Let $h$ be as in (3). Then we know that there is a standard $W=\left(\omega_{i j}\right)$ as in (6.1) such that $L(h)=L(P(W))$. On the other hand, we also know that there is a standard $Y$ as in (6.2) such that $L(h)=f\left(x_{n}^{(j, 0)}, x_{t}^{\omega}\right) L(\widetilde{P}(Y))$ where $f$ is homogeneous. Suppose that $\operatorname{wt}\left(\omega_{i, n}\right)>0$ for $i>i_{0}$. Then for $i>i_{0}$ there is an $\omega_{i^{\prime}, 1}$ such that $\tilde{\omega}_{i, n}<\omega_{i^{\prime}, 1}$ and for $i_{0}<i<j$ we have that $\omega_{i^{\prime}, 1} \leq \omega_{j^{\prime}, 1}$ and that $i^{\prime} \neq j^{\prime}$. It follows that we have $\tilde{\omega}_{i, n}<\omega_{i, 1}$ for $i>i_{0}$.

Theorem 6.5. Let $(V, G)=\left(\ell \mathbb{C}^{n}, \mathrm{SL}_{n}\right)$. Then

$$
p_{\infty}^{*} \mathcal{O}\left(Z_{\infty}\right)=\mathcal{O}\left(V_{\infty}\right)^{G_{\infty}} .
$$

Proof. Let $h \in \mathcal{O}\left(V_{\infty}\right)^{G_{\infty}}$. We may assume that $h$ is homogeneous of a fixed weight. By Corollary 6.4 $L(h)=L(P(W))$ where in the $i$ th row of $W$ we have $\omega_{i, 1}>\tilde{\omega}_{i, n}$ if $\operatorname{wt}\left(\omega_{i, n}\right)>$ 0 . Whenever we have $\omega_{i, 1}>\tilde{\omega}_{i, n}$ we have that $\omega_{i, 1}<\cdots<\omega_{i, n}$ is of the form $\left(j_{1}, k_{1}\right)<\cdots<$ $\left(j_{n}, k_{n}\right)$ where $k_{1}=k_{n}$ or $k_{n}=k_{1}+1$ and $j_{n}<j_{1}$. In the former case we have a sequence $\left(j_{1}, a\right)<\cdots<\left(j_{n}, a\right)$ where $a>0$ and $j_{1}<\cdots<j_{n}$. In the latter case we have a sequence $\left(j_{b+1}, a\right)<\cdots<\left(j_{n}, a\right)<\left(j_{1}, a+1\right)<\cdots<\left(j_{b}, a+1\right)$ where $j_{1}<\cdots<j_{n}$ and $a \geq 0$. Thus the factor of $L(h)$ corresponding to the $i$ th row of $W$ is $L\left(D^{k}\left(\left[x^{\left(j_{1}, 0\right)}, \ldots, x^{\left(j_{n}, 0\right)}\right]\right)\right)$ where $k$ is the sum of the $k_{j}$. Hence there is a homogeneous element $f$ of $p_{\infty}^{*} \mathcal{O}\left(Z_{\infty}\right)$ of the same weight and degree as $h$ such that $L\left(h-p_{\infty}^{*}(f)\right)>L(h)$. By induction we see that $h \in p_{\infty}^{*} \mathcal{O}\left(Z_{\infty}\right)$.

We have not shown that $p_{\infty}^{*}$ is injective, hence we have not shown that $\mathcal{O}\left(Z_{\infty}\right)$ is reduced. Using techniques as in $\S 5$ one can show that $p_{\infty}^{*}$ is an isomorphism when $n=2$. We now show that this is not the case for $n=3$.

Example 6.6. Let $(V, G)=\left(6 \mathbb{C}^{3}, \mathrm{SL}_{3}\right)$. Let $[a b c]$ denote the determinant $\left[x^{(a, 0)}, x^{(b, 0)}, x^{(c, 0)}\right]$ where $1 \leq a<b<c \leq 6$. Consider the corresponding element $f_{a b c} \in \mathcal{O}(Z)$. Now the generators and relations transform by representations of $\mathrm{SL}_{6}$. The generators transform by $\Lambda^{3}\left(\mathbb{C}^{6}\right)$ and the relations are generated by quadratic expressions which transform by the adjoint representation of $\mathrm{SL}_{6}$. The relations involving all six indices are those fixed by the maximal torus $T$ of $\mathrm{SL}_{6}$ and these span a space of dimension $\operatorname{dim} T=5$. Now consider all the possible terms $f_{a b c} D f_{\text {def }}$ and $\left(D f_{a b c}\right) f_{\text {def }}$ where $\{a, b, c, d, e, f\}=\{1, \ldots, 6\}$. It is easy to see that there are six cases where the pullback of these terms to $\mathcal{O}\left(V_{1}\right)$ contains a nonstandard monomial. Since we only have 5 relations to straighten with, we see that one of the straightening relations in $\mathcal{O}\left(V_{\infty}\right)$ does not come from the relations of $\mathcal{O}(Z)$. Hence $p_{\infty}^{*}$ is not injective. Since $p_{\infty}$ is dominant, the kernel of $p_{\infty}^{*}$ consists of nilpotent elements. Hence $Z_{\infty}$ is not reduced. 


\section{REFERENCES}

[Bat] V. Batyrev, Stringy Hodge numbers of varieties with Gorenstein canonical singularities, Integrable systems and algebraic geometry (Kobe/Kyoto, 1997), 1-32, World Sci. Publishing, River Edge, NJ, 1998.

[BD] A. Beilinson and V. Drinfeld, Chiral Algebras, AMS Colloquium Publications 51, 2004.

[Bor] R. Borcherds, Vertex operator algebras, Kac-Moody algebras and the monster, Proc. Nat. Acad. Sci. USA 83 (1986) 3068-3071.

[Bo] J.-F. Boutot, Singularités rationnelles et quotients par les groupes réductifs, Invent. Math. 88 (1987), no. $1,65-68$.

[Cr] A. Craw, An introduction to motivic integration, Amer. Math. Soc., Providence, (2004), 203-225.

[DLI] J. Denef and F. Loeser, Germs of arcs on singular varieties and motivic integration, Invent. Math. 135 (1999), 201-232.

[DLII] J. Denef and F. Loeser, Geometry on arc spaces of algebraic varieties, European Congress of Mathematics, Vol. I (Barcelona, 2000), 327-348, Progr. Math., 201, Birkhäuser, Basel, 2001.

[E] D. Eck, Invariants of k-jet actions, Houston J. Math. Vol. 10, No. 2 (1984), 159-168.

[EM] L. Ein and M. Mustata, Jet schemes and singularities, Algebraic geometry-Seattle 2005. Part 2, 505546, Proc. Sympos. Pure Math., 80, Part 2, Amer. Math. Soc., Providence, RI, 2009.

[FBZ] E. Frenkel and D. Ben-Zvi, Vertex Algebras and Algebraic Curves, Math. Surveys and Monographs, Vol. 88, American Math. Soc., 2001.

[FLM] I.B. Frenkel, J. Lepowsky, and A. Meurman, Vertex Operator Algebras and the Monster, Academic Press, New York, 1988.

[IK] S. Ishii and J. Kollár, The Nash problem on arc families of singularities, Duke Math. J. 120 (2003), no. 3, 601-620.

[Ka] V. Kac, Vertex Algebras for Beginners, University Lecture Series, Vol. 10. American Math. Soc., 1998

[Kol] E. Kolchin, Differential algebra and algebraic groups, Academic Press, New York 1973.

[Kon] M. Kontsevich, Lecture at Orsay (December 7, 1995).

[Kr] H. Kraft, Geometrische Methoden in der Invariantentheorie, Viehweg Verlag, Braunschweig/Wiesbaden 1984.

[LSS] A. R. Linshaw, G. W. Schwarz, and B. Song, Arc spaces and the vertex algebra commutant problem, Adv. Math. 277 (2015), 338-364.

[Loo] E. Looijenga, Motivic measures, Séminaire Bourbaki, Vol. 1999/2000. Astérisque No. 276 (2002), 267297.

[Lun] D. Luna, Slices étales, Sur les groupes algébriques, Soc. Math. France, Paris, 1973, pp. 81-105. Bull. Soc. Math. France, Paris, Mémoire 33.

[MSV] F. Malikov, V. Schechtman, and A. Vaintrob, Chiral de Rham complex, Comm. Math. Phys, 204, (1999) 439-473.

[MS] F. Malikov and V. Schechtman, Chiral de Rham complex, II, Differential topology, infinitedimensional Lie algebras, and applications, 149-188, Amer. Math. Soc. Transl. Ser. 2, 194, Amer. Math. Soc., Providence, RI, 1999.

[Mu] M. Mustata, Jet schemes of locally complete intersection canonical singularities, Invent. Math. 145 (2001), no. 3, 397-424.

[Na] J. Nash, Arc structure of singularities, Duke Math. J. 81, no. 1, 1995, 31-38.

[Sch] G. W. Schwarz, Representations of simple Lie groups with regular rings of invariants, Invent. Math. 49 (1978), no. 2, 167-191.

[So] B. Song, The global sections of the chiral de Rham complex on a Kummer surface, preprint, arXiv:1312.7386.

[Ve] W. Veys, Arc spaces, motivic integration and stringy invariants, Singularity theory and its applications, 529-572, Adv. Stud. Pure Math., 43, Math. Soc. Japan, Tokyo, 2006.

[We] H. Weyl, The Classical Groups: Their Invariants and Representations, Princeton University Press, 1946.

\section{Department of MAthematics, University of DenVER}

E-mail address: andrew.linshaw@du.edu 
DEPARTMENT OF MATHEMATICS, BRANDEIS UNIVERSITY

E-mail address: schwarz@brandeis.edu

Department of MATHEMATICS, University of SCIENCE AND TECHNOlOgy OF CHINA

E-mail address: bailinso@ustc.edu.cn 